\title{
Temporal Resolution of Uncertainty and Recursive Models of Ambiguity Aversion
}

\section{Citation}

Strzalecki, Tomasz. 2013. "Temporal Resolution of Uncertainty and Recursive Models of Ambiguity Aversion." Econometrica 81, no. 3: 1039-1074.

\section{Published Version}

doi:10.3982/ECTA9619

\section{Permanent link}

http://nrs.harvard.edu/urn-3:HUL.InstRepos:12967691

\section{Terms of Use}

This article was downloaded from Harvard University's DASH repository, and is made available under the terms and conditions applicable to Other Posted Material, as set forth at http:// nrs.harvard.edu/urn-3:HUL.InstRepos:dash.current.terms-of-use\#LAA

\section{Share Your Story}

The Harvard community has made this article openly available.

Please share how this access benefits you. Submit a story.

Accessibility 


\section{ECONOMETRICA}

JOURNAL OF THE ECONOMETRIC SOCIETY

An International Society for the Advancement of Economic Theory in its Relation to Statistics and Mathematics

http://www.econometricsociety.org/

Econometrica, Vol. 81, No. 3 (May, 2013), 1039-1074

TEMPORAL RESOLUTION OF UNCERTAINTY AND RECURSIVE

MODELS OF AMBIGUITY AVERSION

TOMASZ STRZALECKI

Harvard University, Cambridge, MA 02138, U.S.A.

The copyright to this Article is held by the Econometric Society. It may be downloaded, printed and reproduced only for educational or research purposes, including use in course packs. No downloading or copying may be done for any commercial purpose without the explicit permission of the Econometric Society. For such commercial purposes contact the Office of the Econometric Society (contact information may be found at the website http://www.econometricsociety.org or in the back cover of Econometrica). This statement must be included on all copies of this Article that are made available electronically or in any other format. 


\title{
TEMPORAL RESOLUTION OF UNCERTAINTY AND RECURSIVE MODELS OF AMBIGUITY AVERSION
}

\begin{abstract}
BY TOMASZ STRZALECKI ${ }^{1}$
Dynamic models of ambiguity aversion are increasingly popular in applied work. This paper shows that there is a strong interdependence in such models between the ambiguity attitude and the preference for the timing of the resolution of uncertainty, as defined by the classic work of Kreps and Porteus (1978). The modeling choices made in the domain of ambiguity aversion influence the set of modeling choices available in the domain of timing attitudes. The main result is that the only model of ambiguity aversion that exhibits indifference to timing is the maxmin expected utility of Gilboa and Schmeidler (1989). This paper examines the structure of the timing nonindifference implied by the other commonly used models of ambiguity aversion. This paper also characterizes the indifference to long-run risk, a notion introduced by Duffie and Epstein (1992). The interdependence of ambiguity and timing that this paper identifies is of interest both conceptually and practically-especially for economists using these models in applications.
\end{abstract}

KEYWORDS: Ambiguity, preference for early resolution of uncertainty.

\section{INTRODUCTION}

\subsection{Background}

THE CONCEPT OF AMBIGUITY has been studied by economists since the work of Keynes (1921) and Knight (1921). As opposed to risk, where objective probabilities are specified, ambiguity is characterized by the inability of the decision maker to formulate a unique probability or by his lack of trust in any single probability estimate. ${ }^{2}$ As demonstrated by Ellsberg (1961), people often make choices that cannot be justified by a unique probability and are willing to pay a premium to insure against ambiguity. ${ }^{3}$

Ambiguity aversion has been a central topic in decision theory in recent years, leading to many elegant formal models. The seminal contributions of Schmeidler (1989) and Gilboa and Schmeidler (1989), followed by

\footnotetext{
${ }^{1}$ This paper is a revised and extended version of Chapters 7 and 8 of my dissertation at Northwestern University; some of the results were also reported in my job market paper. Part of this research was done while I was visiting the Economic Theory Center at Princeton University, to which I am very grateful for its support and hospitality. I thank Roland Benabou, John Campbell, Eddie Dekel, Mira Frick, Drew Fudenberg, Paolo Ghirardato, Faruk Gul, Yoram Halevy, Peter Klibanoff, Fabio Maccheroni, Morgan McClellon, Massimo Marinacci, Stephen Morris, Sujoy Mukherji, Wolfgang Pesendorfer, Ben Polak, Tom Sargent, Todd Sarver, Uzi Segal, and Marciano Siniscalchi for very helpful discussions and suggestions. I am very grateful to a co-editor and three anonymous referees for their insightful and helpful comments. All errors are mine.

${ }^{2}$ In this paper, the word "uncertainty" is an umbrella term for both risk and ambiguity.

${ }^{3}$ Ellsberg only considered thought experiments, but such behavioral patterns were found in experimental studies; see Camerer and Weber (1992) and Halevy (2007) and references therein.
} 
Maccheroni, Marinacci, and Rustichini (2006a) and others, captured the idea that beliefs are not well specified by using capacities and sets of probability measures in the representations of preferences. Later contributions focused on differential attitudes toward risk and ambiguity by using otherwise standard expected utility representations (e.g., Neilson (1993), Klibanoff, Marinacci, and Mukerji (2005), and Ergin and Gul (2009)).

Recent theoretical and applied work involves dynamic models of ambiguity with a recursive formulation

$$
V_{t}=u\left(c_{t}\right)+\beta I\left(V_{t+1}\right),
$$

where uncertainty resolves over time and ambiguity aversion is captured by a certainty equivalent $I$ that is used in each period to assess uncertain continuation values. ${ }^{4}$ This formulation nests the standard model of discounted expected utility as a special case of a linear certainty equivalent $E$ :

$$
V_{t}=u\left(c_{t}\right)+\beta E\left(V_{t+1}\right) .
$$

In general, in situations where uncertainty does not resolve in one shot, agents may distinguish between prospects based on the time at which their uncertainty resolves. However, the standard model of expected discounted utility (2) is separable across both states and time periods, and, therefore, exhibits such indifference to timing. ${ }^{5}$ Recursive models that do exhibit a preference for temporal resolution were first formally studied in the context of risk by Kreps and Porteus (1978), and subsequently were extended and applied to asset pricing; see, for example, Epstein and Zin (1989, 1991), Weil (1989, 1990), and Tallarini (2000), among others. Instead of using standard discounting, these models relax time-separability by using a nonlinear aggregator of the utility of the present consumption and of the certainty equivalent of the continuation value

$$
V_{t}=W\left(c_{t}, E\left(V_{t+1}\right)\right) .
$$

Under the expected utility certainty equivalent, $E$, the nonlinear aggregator $W$ captures the attitude toward temporal resolution of uncertainty, which depends on the curvature of $W$ in its second argument.

\footnotetext{
${ }^{4}$ Models in this class have recently been applied to questions in finance and macroeconomics; see Epstein and Wang (1994), Maenhout (2004), Chen and Epstein (2002), Karantounias, Hansen, and Sargent (2012), Kleshchelski and Vincent (2009), Ju and Miao (2012), Collard, Mukerji, Sheppard, and Tallon (2011), Barillas, Hansen, and Sargent (2009), Li and Tornell (2008), Chen, Ju, and Miao (2009), Benigno and Nisticò (2009), Ilut (2012), and Drechsler (2009).

${ }^{5}$ In settings where actions can be taken after receiving information, early resolution of uncertainty provides decision value to the agent and even the standard model of expected discounted utility exhibits a preference for early resolution. The standard references include Blackwell (1953) and Spence and Zeckhauser (1972). In contrast, this paper focuses on the intrinsic value of information, which arises even in settings with no intermediate decisions.
} 
In addition to preference for temporal resolution, another feature of preferences present in recursive models is aversion to long-run consumption risk. ${ }^{6}$ Duffie and Epstein (1992) observed that discounted expected utility is insensitive to the correlation of payoffs across time periods. For example, it is indifferent between a consumption plan that delivers an independent and identically distributed (i.i.d.) sequence of equiprobable payoffs of $\$ 0$ and $\$ 1$ and another plan that delivers either $\$ 0$ forever or $\$ 1$ forever, with equal ex ante probabilities. On the other hand, models like (3) typically exhibit sensitivity to long-run risk: a feature that underlies much of the recent literature on asset pricing (e.g., Bansal and Yaron (2004), Hansen, Heaton, and Li (2008), and Bansal, Kiku, and Yaron (2012)).

Departures from expected utility and their relation to temporal attitudes have been studied in the context of risk (when probabilities are given). Chew and Epstein (1989) showed that in the class of nonexpected utility preferences, $E$ is the only certainty equivalent that guarantees indifference to temporal resolution. Grant, Kajii, and Polak (2000) strengthened this result by showing that within a fairly large class of preferences, $E$ is the only certainty equivalent that guarantees a (weak) preference for early resolution. These results mean that when probabilities are given, there is not enough flexibility to model separately the attitudes toward temporal resolution and the attitudes toward uncertainty other than expected utility. Under objective risk, neutrality toward timing (or preference for early resolution) cannot be combined with nonexpected utility preferences: any certainty equivalent other than $E$ necessarily produces a nonuniform attitude to temporal resolution.

\subsection{Overview of Results}

This paper studies choice under uncertainty, when probabilities are not a part of the description of alternatives, and formulates the analogues of the notions of preference for earlier resolution of uncertainty and aversion to longrun risk in this framework. The main finding is that under uncertainty, preferences are more flexible than under risk and it is possible to model temporal attitudes separately from the attitudes toward ambiguity, although the class of models that permit such separation is limited. Theorem 1 shows that the only model that exhibits indifference to timing is the maxmin expected utility (MEU) model of Gilboa and Schmeidler (1989), which strictly includes the class of expected utility preferences. Moreover, in contrast to the case of objective risk, Theorems 2 and 4 show that many familiar models of ambiguity aversion display a preference for earlier resolution of uncertainty. Similarly, Theorem 6 and Corollary 3 show that all models of ambiguity commonly used

\footnotetext{
${ }^{6}$ This paper uses the (now standard) term "long-run risk" in all situations of uncertainty, regardless of whether the probabilities are known.
} 
in applications exhibit long-run risk sensitivity, MEU being again the "knifeedge" case of indifference.

These results mean that models of ambiguity aversion are more flexible than models of nonexpected utility under objective risk, as they permit a separation between the timing and uncertainty attitudes that is not possible in the world of risk. Such a separation is useful in applications since nonlinear certainty equivalents can be combined with neutrality toward timing. The possibility of modeling uncertainty attitudes separately from attitudes toward temporal resolution helps one to understand the implications of departing from the expected utility assumption on economic variables such as asset prices, returns, and volatility. Such understanding is not possible in models of objective risk, which entangle these two attitudes and do not allow for changing them separately.

However, these results also mean that models of ambiguity aversion other than MEU lead to timing nonindifference and long-run risk sensitivity even with a linear time aggregator, that is, with standard discounting. Thus, models of ambiguity aversion generally suffer from problems similar to models of objective risk. An implication of this finding is that ambiguity aversion cannot be varied independently without making implicit assumptions about the temporal attitudes, preventing the full separation between these two dimensions of preference.

An extreme case of such lack of separation is the multiplier preferences of Hansen and Sargent (2001), where there is a one-to-one relationship between the degree of ambiguity aversion and the temporal attitudes: preferences with a nonlinear certainty equivalent $I$ have an alternative representation with a linear certainty equivalent $E$ and a nonlinear aggregator $W$. Section 5 studies the general class of reducible preferences where all the nonindifference to timing embodied in $I$ can be captured, in an alternative representation, by an appropriately chosen $W$. Theorem 5 characterizes the class of reducible preferences; this class turns out to be rather small and does not exhaust all models of ambiguity aversion used in applications. Thus emerges a three-way classification: (i) discounted MEU preferences (which are indifferent to timing), (ii) reducible preferences (where $I$ and $W$ are exact "substitutes"), and (iii) irreducible preferences (where $I$ and $W$ are "substitutes," in light of Theorem 1 , but not exact substitutes).

The interdependence of ambiguity and timing that this paper identifies is of interest both conceptually and practically, especially for economists using these models in applications, because it means that the modeling choices that are being made in the domain of ambiguity attitudes influence the set of modeling choices that remain available in the domain of timing attitudes. The results of this paper are qualitative: MEU preferences are indifferent to timing and all other preferences are not; reducible preferences can be reduced to the standard case by an appropriate choice of $W$ and irreducible preferences cannot. A quantitative assessment of the strength of the timing nonindifference in non-MEU preferences would be helpful to understand the importance of 
these effects in practice. For example, in any given model of ambiguity, the calibrated parameters imply a certain ambiguity premium as well as a certain timing premium. ${ }^{7}$ In non-MEU models, these two premiums cannot be varied independently: in reducible preferences, there is a one-to-one relationship between them; the degree to which they are related in irreducible preferences depends on the model in question and on the calibrated parameter values.

The interdependence of ambiguity and timing attitudes in non-MEU models also raises questions about their explanatory power. The preference for earlier resolution of uncertainty and aversion to long-run risk underlie much of recent work on asset pricing. ${ }^{8}$ Given that many applications of ambiguity address the same phenomena, their implied temporal preference makes it hard to assess the added explanatory power of ambiguity aversion because it is not possible to determine whether the predictions of the model about economic variables of interest are driven by ambiguity aversion or by temporal attitudes. Thus, caution is needed in attributing these effects and interpreting results of nonMEU models in applied work. ${ }^{9}$

This paper proceeds as follows: Section 2 defines static ambiguity averse preferences; Section 3 defines discounted ambiguity preferences and the notion of preference for earlier resolution of uncertainty; Section 4 studies the relationship between the attitudes toward ambiguity and timing in models with a linear time aggregator; Section 5 examines the extent to which a separation between ambiguity and nonlinear aggregation can be obtained in non-MEU models; Section 6 studies aversion to long-run risk; finally, Section 7 compares these results to the known results for choice under objective risk.

\section{STATIC MODELS OF AMBIGUITY ATTITUDES}

Let $S$ be the set of states of nature, let $\Sigma$ be an algebra of events, and let $X$ be a set of consequences, assumed to be a convex subset of a real vector space. An act is a $\Sigma$-measurable simple function $f: S \rightarrow X$; the set of acts is denoted $\mathcal{F}$.

\footnotetext{
${ }^{7}$ Epstein, Farhi, and Strzalecki (2013) defined the timing premium in the context of the Epstein-Zin utility and showed that the value of this premium is high even for reasonable parameter values. Computing the magnitude of this premium for models of ambiguity and comparing it with the premium implied by models of pure risk would be helpful in guiding modeling choices and directing attention toward models that imply reasonable values. An experimental investigation of the magnitude of this premium would also be of interest.

${ }^{8}$ In these models, the preference for earlier resolution of uncertainty and aversion to long-run risk are linked in a one-to-one fashion to the wedge between risk aversion and the intertemporal elasticity of substitution. For this reason, the explanatory power of these models in the context of asset pricing may be seen as coming either from the differential attitudes or from the preference for earlier resolution of uncertainty, and is largely a matter of interpretation.

${ }^{9}$ This paper does not settle this issue, but it does outline the basic modeling trade-off by classifying the dynamic ambiguity models into the aforementioned categories. As mentioned above, the exact degree of interdependence depends on the parameter calibrations used; the quantification of its magnitude is left for future work.
} 
Let $B_{0}(\Sigma)$ denote the set of all real-valued $\Sigma$-measurable simple functions and let $B_{0}(\Sigma, K)$ be the set of all such functions that take values in some set $K \subseteq \mathbb{R}$. Let $\Delta(\Sigma)$ be the set of all finitely additive probability measures on $(S, \Sigma)$.

Static preferences studied in this paper are represented by

$$
V(f)=I(u \circ f),
$$

where $u: X \rightarrow \mathbb{R}$ is an affine utility function and $I: B_{0}(\Sigma, u(X)) \rightarrow \mathbb{R}$ is the certainty equivalent that represents the decision maker's "beliefs" by aggregating the utility values over states. It will be maintained throughout that $u$ is unbounded; more specifically, that $u(X)=\mathbb{R}$ or $u(X)=\mathbb{R}_{+}$.

The most basic example of such a functional is that associated with the familiar subjective expected utility (SEU) preferences, where for some probability measure $p \in \Delta(\Sigma)$ the functional is of the form $I(\xi)=\int \xi \mathrm{d} p$ for each $\xi \in B_{0}(\Sigma, u(X))$. Another well known example is the functional associated with Gilboa and Schmeidler's (1989) maxmin expected utility (MEU) preferences, where $I(\xi)=\min _{p \in C} \int \xi \mathrm{d} p$ for some convex and weak ${ }^{*}$-closed set of measures $C \subseteq \Delta(\Sigma) .{ }^{10}$ Other important models include the following:

- Second-order expected utility preferences (Ergin and Gul (2009), Nau (2006), Neilson (1993)), where $I(\xi)=\phi^{-1}\left(\int \phi(\xi) \mathrm{d} p\right)$ for some strictly increasing and concave function $\phi: u(X) \rightarrow \mathbb{R}$.

- Smooth ambiguity preferences (Klibanoff, Marinacci, and Mukerji (2005), Seo (2009); see also Segal (1987)), where

$$
I(\xi)=\phi^{-1}\left(\int_{\Delta(\Sigma)} \phi\left(\int \xi \mathrm{d} p\right) \mathrm{d} \mu(p)\right)
$$

for some strictly increasing and concave function $\phi: u(X) \rightarrow \mathbb{R}$ and a Borel probability measure $\mu$ on $\Delta(\Sigma)$.

- Variational preferences (Maccheroni, Marinacci, and Rustichini (2006a)), where $I(\xi)=\min _{p \in \Delta(\Sigma)} \int \xi \mathrm{d} p+c(p)$ for a convex and weak*-lower semicontinuous function $c: \Delta(\Sigma) \rightarrow[0, \infty]$.

- Multiplier preferences (Hansen and Sargent (2001), Strzalecki (2011)) with $I(\xi)=\min _{p \in \Delta(\Sigma)} \int \xi \mathrm{d} p+\theta R(p \| q)$, where $R(p \| q)$ is the relative entropy of $p$ with respect to some fixed countably additive and nonatomic measure $q \in \Delta(\Sigma)$, and $\theta \in(0, \infty]$ is a parameter.

- Confidence preferences (Chateauneuf and Faro (2009)), defined for $u(X)=\mathbb{R}_{+}$, where for some quasiconcave and weak*-upper semicontinuous function $\varphi: \Delta(\Sigma) \rightarrow[0,1]$ and a parameter $\alpha \in(0,1), I(\xi)=$ $\min _{\{p \in \Delta(\Sigma) \mid \varphi(p) \geq \alpha\}} \frac{1}{\varphi(p)} \int \xi \mathrm{d} p .^{11}$

\footnotetext{
${ }^{10} \mathrm{An}$ important subclass of MEU are Choquet expected utility preferences (Schmeidler (1989)), where $I(\xi)=\int \xi \mathrm{d} v$ for some convex capacity $v: \Sigma \rightarrow[0,1]$.

${ }^{11}$ An extension of these preferences to the case of $u(X)=\mathbb{R}$ was studied by Cerreia-Vioglio, Maccheroni, Marinacci, and Montrucchio (2011, Theorem 21).
} 
All of these examples feature a functional $I$ that is continuous (in the supnorm topology), monotonic (i.e., $I(\xi) \geq I(\zeta)$ whenever $\xi(s) \geq \zeta(s)$ for all $s \in S)$, normalized $(I(k)=k$ for all $k \in u(X)$, interpreted as constant functions), and quasiconcave $(I(\alpha \xi+(1-\alpha) \zeta) \geq \min \{I(\xi), I(\zeta)\})$. The last property corresponds to the famous uncertainty (or ambiguity) aversion axiom of Schmeidler (1989), which postulates that the decision maker does not like variability of payoff across states.

Preferences that can be represented by a belief functional $I$ with these four properties are called uncertainty averse preferences. ${ }^{12}$ This representation of preferences makes it convenient to study attitudes toward ambiguity. A decision maker has constant absolute ambiguity aversion ${ }^{13}$ if $I(\xi+k)=I(\xi)+k$ for all $k \in u(X)$ and $\xi, \xi+k \in B_{0}(\Sigma, u(X))$. The subclass of uncertainty averse preferences with this property is precisely the class of variational preferences. Similarly, a decision maker has constant relative ambiguity aversion if $I(b \xi)=b I(\xi)$ for all $b>0$ and $\xi \in B_{0}(\Sigma, u(X))$. When $u(X)=\mathbb{R}_{+}$, the subclass of uncertainty averse preferences with this property is the class of confidence preferences. ${ }^{14}$ All of the above utility representations have been behaviorally characterized by axioms imposed on the preference relation. The results obtained in this paper are stated directly in the language of these representations; however, all the results can be expressed in the language of preferences, a task that will not be undertaken here.

\section{DYNAMICS}

The purpose of this section is to define formally what it means for the decision maker to care about the timing of uncertainty. To do so, a model will be studied where uncertainty is dated by the time of its resolution: in each period, there is a state space $S$, and the payoff at time $t$ may depend on the realization of the period $t$ uncertainty and/or uncertainty that has already resolved in previous periods. This choice domain is an analogue of Kreps and Porteus's (1978) framework of temporal lotteries, with the difference that here uncertainty is subjective and preferences may not be expected utility. ${ }^{15}$ This re-

\footnotetext{
${ }^{12}$ In a recent paper, Cerreia-Vioglio et al. (2011) showed that $I$ is represented by $I(\xi)=$ $\min _{p \in \Delta(\Sigma)} G\left(\int \xi \mathrm{d} p, p\right)$ for some quasiconvex function $G: \mathbb{R} \times \Delta(\Sigma) \rightarrow \mathbb{R}$ that is increasing in its first argument. The results in this paper do not rely on this (very interesting) representation.

${ }^{13}$ See Proposition 3 of Grant and Polak (2013) and also Definition 6 of Klibanoff, Marinacci, and Mukerji (2005).

${ }^{14}$ As the intersection of both classes, MEU preferences are characterized by both properties.

${ }^{15}$ Epstein and Zin (1989), Chew and Epstein (1989), Segal (1990), and Grant, Kajii, and Polak $(1998,2000)$ studied nonexpected utility preferences in the objective risk framework. Section 7 compares those findings to the results obtained here. The domain of consumption plans that is studied in this paper has been used for axiomatization purposes by Sarin and Wakker (2000), Epstein and Schneider (2003a, 2003b), and Maccheroni, Marinacci, and Rustichini (2006b), among others.
} 
cursive framework is also used in finance and macroeconomics, where in each period, $S$ is the set of possible "shocks."

Formally, time is discrete, $t=0,1, \ldots, T$ with $T$ finite. The set of states of the world is $\Omega=S^{T}$. Information arrival is modeled as the naturally defined filtration $\left\{G_{t}\right\}_{t \in T}$, where $\mathcal{G}_{0}=\{\emptyset, \Omega\}$, and for $t=1, \ldots, T, \mathcal{G}_{t}=\Sigma^{t} \otimes\{\emptyset, S\}^{T-t}$ is the product sigma algebra of $t$ copies of $\Sigma$ and $T-t$ copies of the trivial sigma algebra. Thus, at time $t$, the decision maker knows the realizations of uncertainty up to time $t$, but is ignorant about the future. For any $\omega=\left(s_{1}, \ldots, s_{T}\right)$, let $\omega^{t}=\left(s_{1}, \ldots, s_{t}\right)$ be the history of observations up to time $t$. The consumption plans are modeled as finite-ranged $X$-valued adapted processes $h=\left(h_{0}, h_{1}, \ldots, h_{T}\right)$, where $h_{t}: \Omega \rightarrow X$ is $\mathcal{G}_{t}$-measurable for each $t \leq T$. Let $\mathcal{H}$ denote the set of all consumption plans. The family of relations $\left\{\succsim_{t, \omega}\right\}$ on $\mathcal{H}$ describes the agent's conditional preferences.

\subsection{Attitudes Toward Timing of Resolution of Uncertainty}

This section defines formally the notion of preference for earlier resolution of uncertainty in the domain of subjective uncertainty. The proposed definition is based on the definition of Kreps and Porteus (1978) established in the domain of objective risk (cf. their Theorem 3 and Axiom 5.1). The main idea is to consider a single bet $f$ on $S$ that pays off at time $t+2$. The question is whether at time $t$ the agent prefers to learn about the outcome of $f$ in period $t+1$ or in period $t+2$.

Formally, let $f: S \rightarrow X$ and define a $\mathcal{G}_{t}$-measurable act $\check{f}_{t}: \Omega \rightarrow X$ by $\check{f}_{t}\left(s_{1}, \ldots, s_{T}\right)=f\left(s_{t}\right)$. The act $\check{f}_{t}$ is a "copy" of the bet $f$ that resolves at time $t$, that is, that depends only on the $t$ th component of the state space. Intuitively, given any $f \in \mathcal{F}$, the act $\check{f}_{t}$ is equally uncertain as $\check{f}_{t+1}$, but resolves earlier.

Fix a node $(t, \omega)$ and a consumption plan $h$. Suppose that the only uncertainty that the decision maker faces is about the period $t+2$ payoff, that is, only $h_{t+2}$ is a nondegenerate act. Consider two scenarios. In the first scenario, the uncertainty resolves early, that is, the decision maker learns the realizations of $h_{t+2}$ already in period $t+1$. Formally, let $h_{t+2}=\check{f}_{t+1}$ for some $f \in \mathcal{F}$. In the second scenario, the uncertainty resolves late, that is, the decision maker learns the realizations of $h_{t+2}$ only in period $t+2$. Formally, let $h_{t+2}=\check{f}_{t+2}$ for the same $f \in \mathcal{F}$ as above.

The following definition introduces a binary relation $\geqq_{t}$ on consumption plans that ensures that both plans have no uncertainty other than in period $t+2$ and that uncertainty resolves earlier for the first plan.

DEFINITION 1: For any $h, h^{\prime} \in \mathcal{H}$ and $t \leq T-2$, denote $h \geqq_{t} h^{\prime}$ if and only if there exists $f \in \mathcal{F}$ and $x_{0}, \ldots, x_{t+1}, x_{t+3}, \ldots, x_{T} \in X$ such that $h_{j}=h_{j}^{\prime}=x_{j}$ for all $j \neq t+2, h_{t+2}=\check{f}_{t+1}$, and $h_{t+2}^{\prime}=\check{f}_{t+2}$. 


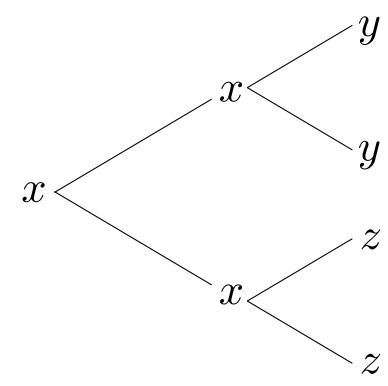

FIGURE 1.-Uncertainty resolves early.

For example, the consumption plan depicted in Figure 1 dominates the consumption plan from Figure 2 according to the relation $\geqq_{0}$. A decision maker whose preferences always respect this order is said to display a preference for earlier resolution of uncertainty.

DEFINITION 2: The family of relations $\left\{\succsim_{t, \omega}\right\}$ exhibits a preference for earlier resolution of uncertainty if and only if for all $h, h^{\prime} \in \mathcal{H}$ and $t \leq T-2$,

$$
h \geqq_{t} h^{\prime} \quad \text { implies } \quad h \succsim_{t^{\prime}, \omega} h^{\prime}
$$

for all $t^{\prime} \leq t$ and $\omega \in \Omega$. The notions of indifference to timing of resolution of uncertainty and strict preference for earlier resolution of uncertainty are defined analogously.

\subsection{Dynamic Models}

\subsubsection{Discounted Uncertainty Averse Preferences}

DEFINITION 3 -Discounted Uncertainty Averse Preferences: A family $\left\{\succsim_{t, \omega}\right\}$ has a discounted uncertainty averse representation with parameters $(\beta, I, u)$

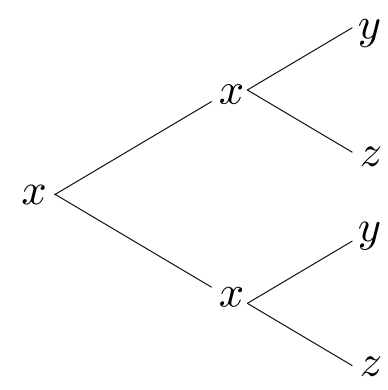

FIGURE 2.-Uncertainty resolves late. 
if it is represented by a family of functionals $V_{t}: \Omega \times \mathcal{H} \rightarrow \mathbb{R}$ defined recursively by $V_{T}(\omega, h)=u\left(h_{T}(\omega)\right)$, and for $t<T$,

$$
V_{t}(\omega, h)=u\left(h_{t}(\omega)\right)+\beta I\left(V_{t+1}(\cdot, h)\right)
$$

where $u: X \rightarrow \mathbb{R}$ is affine, $\beta \in(0,1)$, and $I: B_{0}(\Sigma, u(X)) \rightarrow \mathbb{R}$ is normalized, monotone, continuous, and quasiconcave. ${ }^{16}$

Note that $V_{t+1}(\cdot, h)$ is $\mathcal{G}_{t+1}$-measurable for each $h \in \mathcal{H}$; for this reason, in period $t, V_{t+1}(\cdot, h)$ defines an element of $B_{0}(\Sigma, u(X))$, which represents the uncertainty about the period $t+1$ continuation value that the decision maker faces at period $t$, knowing the history of realizations $\omega^{t}$.

Discounted uncertainty averse preferences include as special cases most of the models used in applications, ${ }^{17}$ but, in general, they allow for more flexible models of ambiguity aversion, as described in Section 2.

An issue that is often discussed in the context of ambiguity averse preferences is that, in general, they violate the regularity properties possessed by the standard model of expected discounted utility: dynamic consistency and consequentialism. ${ }^{18}$ The methods of updating preferences that resolve the trade-off between these two properties include the dynamically consistent but not consequentialist updating rules investigated by Hanany and Klibanoff $(2007,2009)$ and the model of consequentialist but dynamically inconsistent preferences studied by Siniscalchi (2011).

This paper follows a third approach, that of Sarin and Wakker (1998) and Epstein and Schneider (2003b), by restricting the class of events on which updating takes place: the filtration $\left\{\mathcal{G}_{t}\right\}_{t \in \mathcal{T}}$ on the space $\Omega$ is fixed throughout and the only events on which the agent updates belong to $\left\{\mathcal{G}_{t}\right\}_{t \in \mathcal{T}}$. This domain restriction makes room for preferences that are at the same time dynamically consistent and satisfy consequentialism. ${ }^{19}$

\subsubsection{IID Ambiguity}

The effects identified in this paper are also present in a formulation more general than (5), which allows for different certainty equivalents in every period:

$$
V_{t}(\omega, h)=u\left(h_{t}(\omega)\right)+\beta I_{t}\left(\omega^{t}, V_{t+1}(\cdot, h)\right) .
$$

\footnotetext{
${ }^{16}$ The results of this paper hold also under the often used alternate specification of the recursion $V_{t}(\omega, h)=u\left(h_{t}(\omega)\right)+I\left(\beta V_{t+1}(\cdot, h)\right)$.

${ }^{17}$ For example, the recursive maxmin expected utility preferences of Epstein and Schneider (2003b), the recursive smooth ambiguity preferences of Klibanoff, Marinacci, and Mukerji (2009), and the time consistent dynamic variational preferences of Maccheroni, Marinacci, and Rustichini (2006b).

${ }^{18}$ For definitions of these terms in the context of risk, see Machina (1989).

${ }^{19}$ This approach is independent of how the updating rule resolves the trade-off between dynamic consistency and consequentialism on events outside of the event tree determined by the filtration.
} 
However, in this more general model, the attitudes toward timing are confounded with changing beliefs. To see that, observe that given any $f: S \rightarrow X$, the difference between the acts $\breve{f}_{t}$ and $\check{f}_{t+1}$ is twofold. First, these two acts differ in the timing of their resolution. Second, they differ in the extent to which the beliefs about the $t$ th copy of $S$ differ from the beliefs about the $t+1$ th copy of $S$. In the formulation (6), a preference for $\breve{f}_{t}$ over $\breve{f}_{t+1}$ is a result of the intrinsic preference for earlier resolution of uncertainty plus the effect of changing beliefs. ${ }^{20}$ By imposing a "constant beliefs" assumption, known as IID (Independently and Indistinguishably Distributed) ambiguity, ${ }^{21}$ the formulation (5) eliminates this latter effect and isolates the pure attitudes toward timing.

It is worth pointing out that the IID assumption amounts to an assumption on the underlying uncertainty of the environment and not on the consumption process. The consumption process may or may not involve correlation; compare Figures 3 and 4 (which is the reason why the preferences distinguish between them). In fact, just as in the probabilistic environment, a sequence of i.i.d. random variables can be used to construct (almost) any stochastic process: here almost any dependence structure of current on past consumption can be represented. The IID assumption on the state space is needed only to ensure that the notion of early resolution of uncertainty is well defined.

\section{DISCOUNTED PREFERENCES AND TIMING OF RESOLUTION}

This section takes as given a family of discounted uncertainty averse preferences $\left\{\succsim_{t, \omega}\right\}$, defined by expression (5), and examines the relationship between the attitudes toward ambiguity, as described in Section 2, and the attitudes toward timing of resolution of uncertainty, as described in Section 3.1. The main message is that the modeling choices in the domain of ambiguity have strong consequences for the resulting attitudes toward timing. The starkest manifestation of this interdependence is Theorem 1 , which says that the only way to ensure indifference to timing is by using the maxmin expected utility model.

\footnotetext{
${ }^{20}$ This issue does not arise in the model of Kreps and Porteus (1978) because of the objective nature of the probabilities in their formulation. The only difference between the analogues of $\check{f}_{t}$ and $\check{f}_{t+1}$ is the timing of their resolution because their probabilities are objectively the same.

${ }^{21}$ The notion of IID ambiguity was introduced by Chen and Epstein (2002) and Epstein and Schneider (2003a) in the context of the MEU model; it means that the uncertainty that the decision maker faces in period $t$ is identical to the uncertainty in period $t+1$, the only distinguishing property being the timing of their resolution. Intuitively, a decision maker has IID ambiguity if in each period, he faces a new Ellsberg urn; because he observes only one draw from each urn, he cannot make inferences across urns and will not learn his way out of ambiguity, as opposed to observing repeated sampling (with replacement) from the same urn. (The failure of inference in such settings is known in econometrics as the problem of incidental parameters (see, e.g., Neyman and Scott (1948)).)
} 
THEOREM 1: A family of discounted uncertainty averse preferences $\left\{\succsim_{t, \omega}\right\}$ satisfies indifference toward timing of resolution of uncertainty if and only if I is a MEU functional.

The intuitive reason why MEU preferences satisfy indifference to timing is that the worst-case scenario belief is the same irrespective of how far in the future a given act pays off (in other words, the MEU functional has constant relative ambiguity aversion). Similarly, since the MEU functional has constant absolute ambiguity aversion, the preference comparison in Definition 2 is not affected by intermediate payoffs. The proof of Theorem 1 shows that these two properties characterize indifference toward timing; since they also characterize MEU, the result follows.

Theorem 1 implies that assuming any other model of ambiguity aversion results in a family of preferences that exhibits nonindifference to timing. The subsequent theorems in this section examine the structure of the nonindifference implied by models of ambiguity other than MEU. An important, although straightforward, observation is that preference for early resolution is guaranteed by the concavity of $I$.

THEOREM 2: If $\left\{\succsim_{t, \omega}\right\}$ is a family of discounted uncertainty averse preferences represented by a concave functional I, then $\left\{\succsim_{t, \omega}\right\}$ satisfies preference for earlier resolution of uncertainty.

This result implies, in particular, that, as a consequence of their concavity, the variational preferences display a preference for earlier resolution of uncertainty (the only knife-edge case of indifference being the subclass of MEU preferences).

COROLLARY 1: If $\left\{\succsim_{t, \omega}\right\}$ is a family of discounted uncertainty averse preferences where I is variational, then $\left\{\succsim_{t, \omega}\right\}$ satisfies preference for earlier resolution of uncertainty.

The next result shows that when the utility function is unbounded from above and from below (i.e., $u(X)=\mathbb{R}$ ), the variational preferences are the only class of preferences with a concave $I$.

THEOREM 3: Suppose $I: B_{0}(\Sigma) \rightarrow \mathbb{R}$ is concave, monotonic, continuous, and normalized. Then I represents a variational preference.

The intuition behind this result is as follows: concavity and normalization of $I$ imply that $I(\xi+k) \geq I(\xi)+k \in \mathbb{R}$ for all $k$. Since the domain of $I$ is unrestricted, the inequality also has to hold with the reverse sign, implying constant absolute ambiguity aversion, which is the desired conclusion (cf. the definition in Section 2). With a bounded utility, this last step does not have to hold, making room for nonvariational preferences in the class of preferences with a concave representation. 
Theorem 3 points to a modeling restriction that may be of importance in applied work: if a researcher commits to a concave certainty equivalent and an unbounded utility, such as $u(x)=\log x$, then she cannot use any other model than variational preferences.

However, when $u(X)=\mathbb{R}_{+}$, other classes of preferences also admit a concave representation-for example the constant relative ambiguity aversion preferences. Thus, by Theorem 2 , if $u(X)=\mathbb{R}_{+}$, these preferences exhibit a preference for early resolution. By contrast, when $u(X)=\mathbb{R}$, they typically result in nonuniform attitudes toward timing; the only subclass with uniform attitudes is maxmin expected utility preferences.

COROLLARY 2: Suppose that $\left\{\succsim_{t, \omega}\right\}$ is a family of discounted uncertainty averse preferences and I satisfies constant relative ambiguity aversion.

(i) If $u(X)=\mathbb{R}_{+}$, then $\left\{\succsim_{t, \omega}\right\}$ satisfies preference for earlier resolution of uncertainty.

(ii) If $u(X)=\mathbb{R}$ and if $\left\{\succsim_{t, \omega}\right\}$ displays a preference for earlier resolution of uncertainty, then I is MEU.

The results so far show that preferences with concave $I$, such as the variational and the confidence preferences, always exhibit a preference for earlier resolution of uncertainty, while preferences with nonconcave $I$, such as the constant relative ambiguity aversion preferences with $u(X)=\mathbb{R}$, always display a nonuniform attitude toward timing of uncertainty. The next two important classes of preferences are nonconcave, but depending on the underlying function $\phi$, they can either display a preference for earlier resolution of uncertainty or exhibit nonuniform attitudes toward timing. The following two conditions will be used in classifying these cases.

CONDITION 1: There exists $A \geq 0$ such that $-\frac{\phi^{\prime \prime}(a)}{\phi^{\prime}(a)} \in[\beta A, A]$ for all $a \in \mathbb{R}$.

CONDITION 2: $\beta\left[-\frac{\phi^{\prime \prime}(\beta a+k)}{\phi^{\prime}(\beta a+k)}\right] \leq\left[-\frac{\phi^{\prime \prime}(a)}{\phi^{\prime}(a)}\right]$ for all $a, k \in \mathbb{R}_{+}$.

Both conditions require that the curvature of the function $\phi$ (the ArrowPratt coefficient) does not vary too much..$^{22}$ Condition 1 is stronger than Condition 2 . They both permit constant absolute ambiguity aversion ${ }^{23}$; additionally

\footnotetext{
${ }^{22}$ Since the second-order expected utility preferences are reducible (using the terminology of Section 5), the conditions on $\phi$ can be seen as being equivalent to the conditions on the convexity of the aggregator $W$ from Example 1, required by the Kreps-Porteus criterion for preference for earlier resolution of uncertainty. A similar reasoning obtains for the smooth ambiguity preferences.

${ }^{23}$ Constant absolute ambiguity aversion corresponds to the intersection of SOEU with the class of variational preferences, which is precisely the class of the multiplier preferences; see Strzalecki (2011). The fact that those preferences satisfy a preference for earlier resolution of uncertainty follows already from Corollary 1.
} 
Condition 2 permits constant relative ambiguity aversion, that is, $\phi(a)=a^{\gamma}$ for some $\gamma \in(0,1){ }^{24}$

Theorem 4 identifies a subclass of second-order expected utility (SOEU) preferences and smooth ambiguity preferences that displays a preference for earlier resolution of uncertainty.

THEOREM 4: Suppose that $\left\{\succsim_{t, \omega}\right\}$ is a family of discounted uncertainty averse preferences where I is second-order expected utility or smooth ambiguity with a twice differentiable function $\phi$.

(i) If $u(X)=\mathbb{R}$ and if Condition 1 holds, then $\left\{\succsim_{t, \omega}\right\}$ displays a preference for earlier resolution of uncertainty.

(ii) If $u(X)=\mathbb{R}_{+}$and if Condition 2 holds, $\left\{\succsim_{t, \omega}\right\}$ displays a preference for earlier resolution of uncertainty.

REMARK 1: The converse to Theorem 4 holds if $I$ is second-order expected utility. When $I$ is smooth ambiguity, the converse holds under an additional assumption. Theorems 7 and 8 in Appendix A establish these results.

REMARK 2: Note that Theorem 4 together with Theorem 3 imply that the converse to Theorem 2 does not hold. Specifically, suppose that $u(X)=\mathbb{R}$ and $\left\{\succsim_{t, \omega}\right\}$ is a family of discounted uncertainty averse preferences where $I$ is second-order expected utility or smooth ambiguity with $\phi$ that satisfies Condition 1 but is not negative exponential. Theorem 4 implies that $\left\{\succsim_{t, \omega}\right\}$ displays a preference for earlier resolution of uncertainty. However, $\left\{\succsim_{t, \omega}\right\}$ is not convex. To see that, suppose that it is; then, by Theorem $3, I$ is variational which (in light of Theorem 1 of Strzalecki (2011) and Corollary 22 of Cerreia-Vioglio et al. (2011) implies that $\phi$ is negative exponential, a contradiction.

\section{NONLINEAR AGGREGATORS AND REDUCIBLE CERTAINTY EQUIVALENTS}

The results so far demonstrate that MEU is the only certainty equivalent that admits a separation between ambiguity aversion and temporal resolution of uncertainty. MEU is a benchmark of timing indifference since in a model with discounting, the MEU certainty equivalent exhibits timing indifference, while all other certainty equivalents result in timing nonindifference. Since nonneutral timing attitudes can also be obtained by using a nonlinear time aggregator $W$, a natural question is which of the non-MEU certainty equivalents have an alternate representation with a nonlinear $W$ ? A certainty equivalent $I$ is $r e$ ducible if the discounted uncertainty averse preferences represented by $I$ have an alternative representation with a MEU certainty equivalent and a nonlinear aggregator $W$. In other words, $I$ is reducible if all of the timing attitudes

\footnotetext{
${ }^{24}$ Constant relative ambiguity aversion corresponds to the intersection of SOEU with the class of confidence preferences. The fact that those preferences satisfy a preference for earlier resolution of uncertainty follows already from Corollary 2(i).
} 
implicit in $I$ can be explicitly rewritten using $W$. This section obtains a characterization of reducible certainty equivalents.

First, a more general class of preferences is defined by relaxing the standard discounting assumption in expression (5).

DEFINITION 4-Recursive Uncertainty Averse Preferences: A family $\left\{\succsim_{t, \omega}\right\}$ has a recursive uncertainty averse representation with $(W, I, v)$ if it is represented by a family of functionals $V_{t}: \Omega \times \mathcal{H} \rightarrow \mathbb{R}$ defined recursively by $V_{T}(\omega, h)=$ $v\left(h_{T}(\omega)\right)$ and for $t<T$,

$$
V_{t}(\omega, h)=W\left(h_{t}(\omega), I\left(V_{t+1}(\cdot, h)\right)\right),
$$

where $v: X \rightarrow \mathbb{R}$, the aggregator $W: X \times \mathbb{R} \rightarrow \mathbb{R}$ is continuous, strictly increasing, and unbounded in the second argument, and $I: B_{0}(\Sigma) \rightarrow \mathbb{R}$ is normalized, monotone, continuous, and quasiconcave.

These preferences are a natural generalization of those studied in Koopmans (1960), Kreps and Porteus (1978), and Epstein and Zin (1989) to subjective uncertainty. ${ }^{25}$ The discounted preferences defined by (5) correspond to the special case of recursive preferences defined by (7) with $W^{\text {disc }}(x, \gamma)=u(x)+$ $\beta \gamma$ for some affine function $u: X \rightarrow \mathbb{R}$. The model of Kreps and Porteus (1978) has $I^{\mathrm{EU}}(\xi)=\int \xi \mathrm{d} p$. In this model, the standard discounting aggregator $W^{\text {disc }}$ implicit in expression (5) characterizes indifference to timing of resolution of uncertainty, while nonlinear aggregators lead to nonindifference (in particular, convexity of $W$ in the second argument corresponds to the case of preference for earlier resolution).

DEFINITION 5-Reducible Certainty Equivalent: A certainty equivalent $I: B_{0}(\Sigma) \rightarrow \mathbb{R}$ is reducible if and only if a family of discounted uncertainty averse preferences $\left\{\succsim_{t, \omega}\right\}$ with representation ( $W^{\text {disc }}, I, u$ ) has a recursive uncertainty averse representation with $\left(W, I^{\mathrm{MEU}}, v\right)$ for some $\mathrm{MEU}$ certainty equivalent $I^{\mathrm{MEU}}$.

EXAMPLE 1: Consider the second-order expected utility certainty equivalent: $I^{\text {SOEU }}(\xi)=\phi^{-1}\left(\int \phi(\xi(s)) \mathrm{d} q(s)\right)$ and $W^{\text {disc }}(x, \gamma)=u(x)+\beta \gamma$. Theorem 1 implies that such preferences exhibit timing nonindifference; note that this nonindifference may be attributed to the fact that the certainty equivalent does not belong to the MEU class. However, these preferences can be rewritten as Kreps-Porteus preferences with $I^{\mathrm{EU}}(\xi)=\int \xi(s) \mathrm{d} q(s)$ and $W(x, d)=\phi\left(u(x)+\beta \phi^{-1}(d)\right)$ for all $x \in X$ and all $d \in \operatorname{Range}(\phi)$. From this

\footnotetext{
${ }^{25}$ Other subjective extensions have been studied and axiomatized by Hayashi (2005), Klibanoff and Ozdenoren (2007), and Skiadas (1998). Skiadas (1998) also studied attitudes toward timing by assuming that preferences are defined over pairs consisting of a consumption plan and exogenously given information in the form of a filtration to which the consumption plan is adapted. For a continuous time extension, see Lazrak (2004).
} 
point of view, the timing nonindifference may be attributed entirely to the nonlinear aggregator, since the certainty equivalent is (a special case of) MEU.

Example 1 shows that second-order expected utility is reducible. ${ }^{26}$ In general, reducible certainty equivalents result in preferences where $I$ and $W$ are substitutes: timing nonindifference can be attributed either to the curvature of $I$ ( $I$ being outside of the MEU class) or the curvature of $W$ ( $W$ being outside of the discounting class). The next theorem characterizes the class of reducible certainty equivalents.

DEFINITION 6: The functional $I$ is a second-order maxmin expected utility (SOMEU) if and only if there exists a strictly increasing and concave function $\phi: \mathbb{R} \rightarrow \mathbb{R}$ and a convex and weak*-closed set $C \subseteq \Delta(\Sigma)$ such that $I(\xi)=\min _{p \in C} \phi^{-1}\left(\int \phi(\xi) \mathrm{d} p\right)$.

THEOREM 5: A certainty equivalent I is reducible if and only if I is SOMEU.

The class of reducible certainty equivalents characterized by Theorem 5 has two parameters: a set of priors $C$ and a curving function $\phi$. This class generalizes both the MEU preferences (with $\phi$ being an affine function) and secondorder expected utility preferences (with $C$ being a singleton). For such preferences, the timing nonindifference induced by the non-MEU nature of the certainty equivalent can be exactly offset by an appropriate choice of the aggregator. The exact form of the aggregator is given in the following remark.

REMARK 3: If $I$ is reducible, then the recursive representation $\left(W, I^{\mathrm{MEU}}, v\right)$ has $v(x)=\phi(u(x))$ for all $x \in X, I^{\mathrm{MEU}}(\xi)=\min _{p \in C} \int \xi \mathrm{d} p$ for all $\xi \in B_{0}(\Sigma)$, and either (a) $W(x, d)=\phi\left(u(x)+\beta \phi^{-1}(d)\right)$ for all $x \in X$ and all $d \in$ Range $(\phi)$ or (b) there exist $a>0, e, c, b \in \mathbb{R}$ such that $W(x, d)=a \phi(u(x)+$ $\left.\beta \phi^{-1}(d)\right)+b$ for all $x \in X$, all $d \in \operatorname{Range}(\phi)$, and $\phi(r)=e a^{r / c}+\frac{b}{1-a}$ for all $r \in \mathbb{R}$.

\section{AVERSION TO LONG-RUN RISK}

This section studies another behavioral property of preferences: the attitude toward long-run risk. The proposed definition adapts the notions formally introduced by Duffie and Epstein (1992) to the domain of subjective uncertainty.

Consider the following two consumption plans. In the first one, a coin is tossed every period and the payoff in each period depends on that period's coin toss, say $\$ 1$ if heads and $\$ 0$ if tails. This scenario will be referred to as i.i.d. risk or short-run risk. In the second consumption plan, the coin is tossed only once, at the beginning of time, and then either $\$ 1$ or $\$ 0$ is paid forever. This scenario will be referred to as long-run risk. See Figures 3 and 4 . 


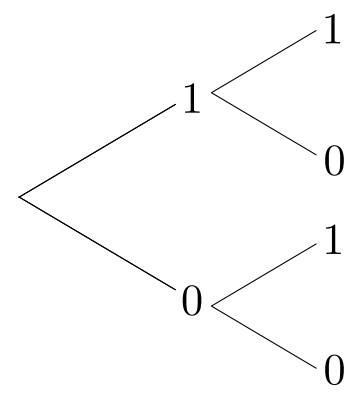

FIGURE 3.-IID risk.

In general, a decision maker may well have different sensitivities to short and long-run shocks; however, an expected discounted utility model forces indifference as a consequence of the separability of the criterion across states and time periods.

As in Section 3.1, for any $f: S \rightarrow X$, define a $\mathcal{G}_{t}$-measurable act $\check{f}_{t}: \Omega \rightarrow X$ by $\check{f}_{t}\left(s_{1}, \ldots, s_{T}\right)=f\left(s_{t}\right)$; that is, the act $\check{f}_{t}$ is a copy of the act $f$ that resolves at time $t$ (i.e., that depends only on the $t$ th component of the state space). The short-run risk (or i.i.d. risk) consumption plan associated with act $f$ is $h_{f}^{\mathrm{SR}}=\left(\check{f}_{0}, \check{f}_{1}, \ldots, \check{f}_{T}\right)$. The long-run risk consumption plan associated with act $f$ is $h_{f}^{\mathrm{LR}}=\left(\check{f}_{0}, \check{f}_{0}, \ldots, \check{f}_{0}\right)$.

DEFINITION 7: The family of relations $\left\{\succsim_{t, \omega}\right\}_{(t, \omega) \in \mathcal{T} \times \Omega}$ exhibits aversion to long-run risk if and only if for all $f \in \mathcal{F}$, the preference $h_{f}^{\mathrm{SR}} \succsim_{0} h_{f}^{\mathrm{LR}}$ holds. The notions of indifference to long-run risk and strict aversion to long-run risk are defined analogously.

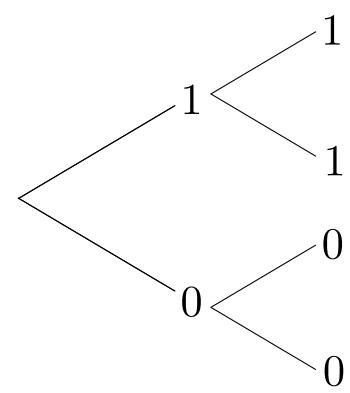

FIGURE 4.--Long-run risk.

\footnotetext{
${ }^{26}$ In particular, the multiplier preferences of Hansen and Sargent (2001) belong to this class by taking $\phi$ to be negatively exponential.
} 
The following results establish the relationship between the attitudes toward ambiguity, as described in Section 2, and the attitudes toward long-run risk, as described in Definition 7. Similarly to the case of timing of resolution, the main message is that the modeling choices in the domain of ambiguity have strong consequences for the resulting attitudes toward long-run risk. The following theorem shows that in the class of preferences with concave representation, all preferences but MEU display a strict aversion to long-run risk.

THEOREM 6: Suppose that $\left\{\succsim_{t, \omega}\right\}$ is a family of discounted uncertainty averse preferences where I is concave and strictly monotone, that is, $I(\xi+k)>I(\xi)$ for all $\xi$ and $k>0$. Then $\left\{\succsim_{t, \omega}\right\}$ satisfies aversion to long-run risk. Moreover, it displays indifference if and only if I is a MEU functional.

The following corollary shows that Theorem 6 covers all utility specifications commonly used in applications.

COROLLARY 3:

(i) Any family $\left\{\succsim_{t, \omega}\right\}$ of dynamic variational preferences displays aversion to long-run risk with indifference if and only if $\left\{\succsim_{t, \omega}\right\}$ is $M E U$.

(ii) Any family $\left\{\succsim_{t, \omega}\right\}$ of confidence preferences with $u(X)=\mathbb{R}_{+}$displays aversion to long-run risk with indifference if and only if $\left\{\succsim_{t, \omega}\right\}$ is $M E U$.

(iii) Any family $\left\{\succsim_{t, \omega}\right\}$ of smooth ambiguity or second-order expected utility preferences with concave $\phi$ that is constant absolute risk aversion (CARA) or constant relative risk aversion $(C R R A)$ displays aversion to long-run risk with indifference if and only if $\left\{\succsim_{t, \omega}\right\}$ is expected utility $(E U)$.

REMARK 4: It is worth noticing that long-run risk involves early resolution of uncertainty, since all future payoffs will be known in period 1 . However, although both variational and confidence preferences exhibit a preference for earlier resolution of uncertainty, they exhibit aversion to long-run risk because the aversion to correlation of payoffs is stronger than the preference for earlier resolution of uncertainty.

\section{COMPARISON TO CHOICE UNDER RISK}

\subsection{Intertemporal Elasticity of Substitution}

The model of Kreps and Porteus (1978) allows for a separation between the elasticity of substitution between states and between time periods. ${ }^{27}$ However, in that model, the difference between these two elasticities is directly related to the strength of the preference for timing of resolution of uncertainty. In other words, the three features-intertemporal elasticity of substitution, elas-

\footnotetext{
${ }^{27}$ This issue has also been studied, among others, by Kihlstrom and Mirman (1974), Selden (1978), and Chew and Epstein (1990) on choice domains, which do not allow for temporal resolution of uncertainty.
} 
ticity of substitution between states, and preference for timing of resolution of uncertainty —are interdependent; roughly speaking, knowing two of them is sufficient to determine the third. For this reason, the Kreps-Porteus model may be seen as restrictive because it does not allow enough freedom to specify the three parameters independently.

To see this, suppose that $u(x)=x^{\alpha}$ for some $\alpha \in(0,1)$. Consider first a discounted second-order expected utility model, with $\phi(x)=x^{\rho}$ for some $\rho \in(0,1]$. This constitutes a (subjective) analogue of the Kreps-Porteus model. In this model, the intertemporal elasticity of substitution is equal to $(1-\alpha)^{-1}$, whereas the elasticity of substitution between states is equal to $(1-\alpha \rho)^{-1}$. As long as these two are different, that is, as long as $\rho \neq 1$, the decision maker will not be indifferent toward timing of resolution of uncertainty. ${ }^{28}$

From this point of view, dynamic ambiguity models allow more flexibility. In particular, a discounted MEU model allows for a separation of the three features. Indifference to timing is guaranteed by Theorem 1, while the intertemporal elasticity of substitution is $(1-\alpha)^{-1}$, which is not identically equal to the elasticity of substitution between states. The latter varies between zero and $(1-\alpha)^{-1}$, depending on the act at which it is computed. This shows that it is possible to drive a wedge between the two elasticities without forcing timing nonindifference.

\subsection{Timing Attitudes}

Dynamic models of ambiguity may be seen as more flexible than those of risk for yet another reason. As Theorem 1 of Chew and Epstein (1989) shows, when preferences are defined over lotteries rather than acts, indifference to timing implies that the certainty equivalent (the counterpart of the functional $I$ in their model) has to be expected utility. In contrast, Theorem 1 of this paper shows that in the domain of acts, the class of preferences indifferent to timing is larger: it is precisely MEU.

Even more restrictive is the fact that most of the known departures from expected utility in the risk domain induce a nonuniform attitude toward timing (much like the constant relative ambiguity aversion preferences of Corollary 2(ii)). Proposition 1 of Grant, Kajii, and Polak (2000) shows that (if preferences are rank-dependent or satisfy betweenness) expected utility is a necessary consequence of preference for earlier resolution of uncertainty. ${ }^{29} \mathrm{In}$ contrast, Theorems 1-4 of this paper show that in the domain of acts, the class

\footnotetext{
${ }^{28}$ According to Theorem 1, the decision maker displays an indifference to the timing of resolution of uncertainty if and only if $I$ is MEU. The only intersection of MEU and second-order expected utility preferences is expected utility preferences (i.e., $\rho=1$ ).

${ }^{29}$ More precisely, Proposition 1 of Grant, Kajii, and Polak (2000) as well as Theorem 1 of Chew and Epstein (1989) allow for the certainty equivalent in each time period to differ and show that all but the first or last certainty equivalents have to be EU. These results imply the above statements in the context of a model with a constant certainty equivalent, like the one here.
} 
of such preferences is larger: it includes all variational and confidence preferences, as well as certain second-order expected utility and smooth ambiguity preferences.

An illustrative case in point is the rank-dependent utility (RDU) of Quiggin (1982) and Yaari (1987) in which probability distributions are distorted by a transformation function. When the preferences are defined on acts, assuming that the probability transformation function is convex, this model reduces to Choquet expected utility with a convex capacity-a special case of MEU—and thus satisfies timing indifference. On the other hand, when the preferences are defined on lotteries, the aforementioned results imply nonindifference (and nonuniform attitude) to timing.

It should be stressed that these differences are not a consequence of the conceptual distinction between risk and ambiguity, but rather they are caused by the dissimilarity of the two choice domains. In particular, the definition of early resolution (relation $\geqq$ ) in the subjective domain is less restrictive than in the model with objective probabilities. This is caused by the fact that in the objective setting, earlier resolution is defined through probability mixtures of lotteries, while in the subjective setting the-less flexible-eventwise mixtures are used. For this reason, although RDU does not preserve the indifferences to timing for all comparable pairs of temporal lotteries in the objective domain, it does so in the subjective domain because there are fewer such $\geqq$-comparable pairs.

\subsection{Subjective versus Objective Uncertainty}

The focus of this paper is the preference for the timing of resolution of subjective uncertainty. However, in environments where both subjective uncertainty and objective uncertainty (or risk) are present, it is possible for preferences to display differential attitudes to the resolution of both types of uncertainty. In fact, most models studied in this paper will have this property.

The choice domain of this paper can be seen as capturing risk if the set $X$ is interpreted as the set of objective probability distributions on some primitive set of prizes; however, the preferences for early resolution of risk cannot be captured by the domain, as defined, because the probability distributions represented by $X$ are on final outcomes instead of continuation acts. However, under a straightforward extension of the domain, studying attitudes to both types of resolution is possible..$^{30}$

On such an extended choice domain, the discounted uncertainty averse preferences (as in Section 4) involve nonindifference to the timing of uncertainty

\footnotetext{
${ }^{30}$ If the time horizon is finite, as in this paper, defining such a domain involves a simple finitely recursive definition. For an infinite horizon, the definition is more involved and comes at the cost of assuming a finite state space (see, e.g., Hayashi (2005)).
} 
(as long as $I$ is not MEU-the case axiomatized by Hayashi (2005)), but indifference to the timing of risk. More generally, in a recursive version of the model (as in Section 5), the preferences would exhibit different preferences for the timing of the resolution of uncertainty. ${ }^{31}$ In such models, the preference for the timing of the resolution of risk is captured by the aggregator $W$, while the preference for the timing of the resolution of subjective uncertainty is induced by the ambiguity aversion certainty equivalent $I$ in addition to the direct effect coming from the aggregator $W$.

In a related paper, Ergin and Sarver (2010) studied preferences for the timing of the resolution of objective risk with a different choice domain, equal to lotteries over menus of lotteries over the final outcomes. The preferences they study are von Neumann-Morgenstern (vNM) over the outer layer of lotteries, but the preferences over menus violate strategic rationality; following Kreps (1979) and Dekel, Lipman, and Rustichini (2001), a subjective state space is derived from preferences. Ergin and Sarver (2010) associated the preference for later resolution of risk with there being a malevolent nature that is minimizing the agent's expected utility over some set of probabilities, a representation reminiscent of ambiguity aversion. In fact, analogous effects are present in models with an objective state space and two layers of objective risk, like those of Anscombe and Aumann (1963) and, more recently, Seo (2009). Under vNM over the outer layer of lotteries, these effects manifest themselves as a preference for mixing acts ex post (after the realization of the state), rather than ex ante: a phenomenon captured by the uncertainty aversion axiom of Schmeidler (1989). In models with an objective state space, this feature arises because mixing acts ex post provides hedging, which is desired under ambiguity aversion. In the Ergin and Sarver (2010) model, this feature is present even under objective risk, since any given single lottery in their model can be seen as a nondegenerate act with respect to the subjective state space.

\section{APPENDIX A: ADDITIONAL RESULTS}

The following theorem presents a converse to Theorem 4 for second-order expected utility preferences.

THEOREM 7: Suppose that $\left\{\succsim_{t, \omega}\right\}$ is a family of discounted uncertainty averse preferences where I is second-order expected utility with a twice differentiable function $\phi$.

(i) If $u(X)=\mathbb{R}$ and it displays a preference for earlier resolution of uncertainty, then Condition 1 holds.

(ii) If $u(X)=\mathbb{R}_{+}$and it displays a preference for earlier resolution of uncertainty, then Condition 2 holds.

\footnotetext{
${ }^{31}$ A recent paper by Hayashi and Miao (2011) formulates and axiomatizes a special case of such preferences, based on Klibanoff, Marinacci, and Mukerji (2005).
} 
The following theorem presents a partial converse to Theorem 4 for smooth ambiguity preferences. The assumption under which the converse is established implies that there are more states in $S$ than there are measures in the support of $\mu$. This condition also implies the full rank condition of Klibanoff, Marinacci, and Mukerji (2009), which yields a characterization of Bayesian updating in their model.

ASSUMPTION 1: $S$ is finite with cardinality $n$ and the support of the measure $\mu$ is finite with cardinality $m$. For $j=1, \ldots, m$, let each measure $p_{j} \in \operatorname{supp} \mu$ be represented as a row vector in $\mathbb{R}^{n}$ and let $M$ be an $m \times n$ matrix of those vectors stacked on top of each other. The matrix M has rank $m$.

THEOREM 8: Suppose that $\left\{\succsim_{t, \omega}\right\}$ is a family of discounted uncertainty averse preferences where I is smooth ambiguity preferences with a twice differentiable function $\phi$ that satisfies Assumption 1.

(i) If $u(X)=\mathbb{R}$ and it displays a preference for earlier resolution of uncertainty, then Condition 1 holds.

(ii) If $u(X)=\mathbb{R}_{+}$and it displays a preference for earlier resolution of uncertainty, then Condition 2 holds.

\section{APPENDIX B: PROOFS}

LEMMA 1: The family $\left\{\succsim_{t, \omega}\right\}$ displays a preference toward earlier resolution of uncertainty if and only if $I(\beta \xi+k) \geq \beta I(\xi)+k$ for all $\xi \in B_{0}(\Sigma, u(X))$ and all $k \in u(X)$. The family $\left\{\succsim_{t, \omega}\right\}$ displays indifference toward timing of resolution of uncertainty if and only if $I(\beta \xi+k)=\beta I(\xi)+k$ for all $\xi \in B_{0}(\Sigma, u(X))$ and all $k \in u(X)$.

Proof: Fix $x_{0}, \ldots, x_{T} \in X$ and $f \in \mathcal{F}$. Let $l:=\beta^{0} u\left(x_{t+3}\right)+\cdots+$ $\beta^{T-t-3} u\left(x_{T}\right)$. Observe that

$$
\begin{aligned}
& V_{t}\left(\omega,\left(x_{0}, \ldots, x_{t+1}, f_{t+1}, x_{t+3}, \ldots, x_{T}\right)\right) \\
& \quad=u\left(x_{t}\right)+\beta I\left(u\left(x_{t+1}\right)+\beta I\left(u\left(f_{t+1}\left(\omega^{t+1}, \cdot\right)\right)+\beta l\right)\right) .
\end{aligned}
$$

Because $f_{t+1}$ is $\mathcal{G}_{t+1}$-measurable, $I\left(u\left(f_{t+1}\left(\omega^{t+1}, \cdot\right)\right)\right)=u\left(f_{t+1}(\omega)\right)=u\left(f\left(s_{t+1}\right)\right)$ for all $\omega \in \Omega$; thus by denoting $\zeta:=u(f)$,

$$
\begin{gathered}
V_{t}\left(\omega,\left(x_{0}, \ldots, x_{t+1}, f_{t+1}, x_{t+3}, \ldots, x_{T}\right)\right) \\
\quad=u\left(x_{t}\right)+\beta I\left(u\left(x_{t+1}\right)+\beta(\zeta+\beta l)\right) .
\end{gathered}
$$

On the other hand,

$$
\begin{aligned}
& V_{t}\left(\omega,\left(x_{0}, \ldots, x_{t+1}, f_{t+2}, x_{t+3}, \ldots, x_{T}\right)\right) \\
& \quad=u\left(x_{t}\right)+\beta I\left(u\left(x_{t+1}\right)+\beta I\left(u\left(f_{t+2}\left(\omega^{t+1}, \cdot\right)\right)+\beta l\right)\right) .
\end{aligned}
$$


Because $f_{t+2}$ does not depend on $\omega_{t+1}, I\left(u\left(f_{t+2}\left(\omega^{t+1}, \cdot\right)\right)\right)=I(u(f))$. Thus,

$$
\begin{gathered}
V_{t}\left(\omega,\left(x_{0}, \ldots, x_{t+1}, f_{t+2}, x_{t+3}, \ldots, x_{T}\right)\right) \\
\quad=u\left(x_{t}\right)+\beta\left(u\left(x_{t+1}\right)+\beta I(\zeta+\beta l)\right) .
\end{gathered}
$$

Suppose $I$ displays a preference for earlier resolution of uncertainty. Then expression (8) is larger than (9) for any choice of $x_{0}, \ldots, x_{T} \in X$ and $\zeta \in B_{0}(\Sigma)$, in particular, such that $u\left(x_{t+1}\right)=k$ and $\zeta=\xi+\beta l$. The converse and the proof of the statement about indifference follow similarly.

Q.E.D.

Lemma 2: Define $B:=\beta+\beta^{2}+\cdots+\beta^{T}$. The family $\left\{\succsim_{t, \omega}\right\}$ displays aversion to long-run risk if and only if $I(\xi+B I(\xi)) \geq I((1+B) \xi)$ for all $\xi \in B_{0}(\Sigma, u(X))$. The family $\left\{\succsim_{t, \omega}\right\}$ displays indifference toward long-run risk if and only if $I(\xi+$ $B I(\xi))=I((1+B) \xi)$ for all $\xi \in B_{0}(\Sigma, u(X))$.

PRoOF: By Definition 7, $\left\{\succsim_{t, \omega}\right\}_{(t, \omega) \in \mathcal{T} \times \Omega}$ exhibits aversion to long-run risk if and only if for all measurable $f: S \rightarrow X$,

$$
V\left(h_{f}^{\mathrm{SR}}\right) \geq V\left(h_{f}^{\mathrm{LR}}\right) .
$$

Fix $\xi:=u(f)$ and observe that $V\left(h_{f}^{\mathrm{SR}}\right)=V\left(\check{f}_{0}, \check{f}_{1}, \ldots, \check{f}_{T}\right)=I(\xi+B I(\xi))$, while $V\left(h_{f}^{\mathrm{LR}}\right)=V\left(\check{f}_{0}, \check{f}_{0}, \ldots, \check{f}_{0}\right)=I((1+B) \xi)$. Thus, $(10)$ holds if and only if $I(\xi+B I(\xi)) \geq I((1+B) \xi)$ for all $\xi \in B_{0}(\Sigma, u(X))$.

The analogous proof holds with equality for indifference.

Q.E.D.

Lemma 3: Let $\Phi:=\phi(u(X))$ and, for each $k \in u(X)$, define $F_{k}: \Phi \rightarrow \mathbb{R}$ by $F_{k}(\gamma)=\phi\left(\beta \phi^{-1}(\gamma)+k\right)$. Suppose that $\phi$ is twice differentiable. Then $F_{k}$ is convex for each $k \in u(X)$ if and only if $\beta\left[-\frac{\phi^{\prime \prime}(\beta a+k)}{\phi^{\prime}(\beta a+k)}\right] \leq\left[-\frac{\phi^{\prime \prime}(a)}{\phi^{\prime}(a)}\right]$ for all $a, k \in$ $u(X)$.

Proof: Because $\phi$ is twice differentiable, $\phi^{-1}$ is twice differentiable. Convexity of $F_{k}$ for each $k \in u(X)$ is equivalent to

$$
F_{k}^{\prime \prime}(\gamma) \geq 0 \text { for all } k \in u(X) \text { and } \gamma \in \Phi .
$$

A direct computation reveals that

$$
\begin{aligned}
F_{k}^{\prime \prime}(\gamma)= & \phi^{\prime \prime}\left(\beta \phi^{-1}(\gamma)+k\right)\left[\frac{\beta}{\phi^{\prime}\left(\phi^{-1}(\gamma)\right)}\right]^{2} \\
& -\phi^{\prime}\left(\beta \phi^{-1}(\gamma)+k\right) \frac{\beta \phi^{\prime \prime}\left(\phi^{-1}(\gamma)\right)}{\left[\phi^{\prime}\left(\phi^{-1}(\gamma)\right)\right]^{3}} .
\end{aligned}
$$

Thus, $F_{k}^{\prime \prime}(\gamma) \geq 0$ if and only if $\beta\left[-\frac{\phi^{\prime \prime}\left(\beta \phi^{-1}(\gamma)+k\right)}{\phi^{\prime}\left(\beta \phi^{-1}(\gamma)+k\right)}\right] \leq\left[-\frac{\phi^{\prime \prime}\left(\phi^{-1}(\gamma)\right)}{\phi^{\prime}\left(\phi^{-1}(\gamma)\right)}\right] \quad$ Q.E.D. 
LEMMA 4: Let $u(X)=\mathbb{R}$ and $\Phi:=\phi(\mathbb{R})$, and for each $k \in \mathbb{R}$, define $F_{k}: \Phi \rightarrow$ $\mathbb{R}$ by $F_{k}(\gamma)=\phi\left(\beta \phi^{-1}(\gamma)+k\right)$. Suppose that $\phi$ is twice differentiable. Then $F_{k}$ is convex for each $k \in \mathbb{R}$ if and only if there exists $A \geq 0$ such that $-\frac{\phi^{\prime \prime}(a)}{\phi^{\prime}(a)} \in[\beta A, A]$ for all $a \in \mathbb{R}$.

ProOF: Let $a, b \in \mathbb{R}$. Define $k:=b-\beta a$. By Lemma 3 , convexity of $F_{k}$ is equivalent to

$$
\beta\left[-\frac{\phi^{\prime \prime}(b)}{\phi^{\prime}(b)}\right] \leq\left[-\frac{\phi^{\prime \prime}(a)}{\phi^{\prime}(a)}\right] \text { for all } a, b \in \mathbb{R}
$$

It is immediate that condition (12) is implied if there exists $A \geq 0$ such that $-\frac{\phi^{\prime \prime}(a)}{\phi^{\prime}(a)} \in[\beta A, A]$ for all $a \in \mathbb{R}$. Conversely, let $A:=\sup _{b \in \mathbb{R}}\left[-\frac{\phi^{\prime \prime}(b)}{\phi^{\prime}(b)}\right]$. The number $A$ is finite, because otherwise condition (12) is violated by fixing $a$ and letting the left hand side diverge. Let $A^{\prime}:=\inf _{a \in \mathbb{R}}\left[-\frac{\phi^{\prime \prime}(a)}{\phi^{\prime}(a)}\right]$. If $A^{\prime}<\beta A$, then find two real numbers such that $A^{\prime}<l^{\prime}<l<\beta A$. There exist $a, b \in \mathbb{R}$ with $\left[-\frac{\phi^{\prime \prime}(a)}{\phi^{\prime}(a)}\right]<l^{\prime}$ and $l \beta^{-1}<\left[-\frac{\phi^{\prime \prime}(b)}{\phi^{\prime}(b)}\right]$. Thus, $\left[-\frac{\phi^{\prime \prime}(a)}{\phi^{\prime}(a)}\right]<l^{\prime}<l<\beta\left[-\frac{\phi^{\prime \prime}(b)}{\phi^{\prime}(b)}\right]$. Contradiction.

Q.E.D.

Lemma 5: Suppose $K \in\left\{\mathbb{R}_{+}, \mathbb{R}\right\}$ and $I: B_{0}(\Sigma, K) \rightarrow \mathbb{R}$ is concave and normalized, and there exists $\alpha \in(0,1)$ such that $I(\alpha \xi)=\alpha I(\xi)$ for all $\xi \in B_{0}(\Sigma, K)$. Then I is positively homogeneous.

PROOF: By concavity and normalization, it follows that $I(b \xi)=I(b \xi+(1-$ b)0) $\geq b I(\xi)+(1-b) I(0)=b I(\xi)$ for all $\xi \in B_{0}(\Sigma, K)$ and all $b \in(0,1)$.

Suppose, toward contradiction, that there exist $a \in(0,1)$ and $\xi \in B_{0}(\Sigma, K)$ such that $I(a \xi)>a I(\xi)$. Observe that $I\left(\alpha^{n} \xi\right)=I\left(\alpha \alpha^{n-1} \xi\right)=\alpha I\left(\alpha^{n-1} \xi\right)=$ $\cdots=\alpha^{n} I(\xi)$ for any $n \in \mathbb{N}$. Choose $n$ such that $\alpha^{n}<a$. For this $n$, it follows that $\alpha^{n} I(\xi)=I\left(\alpha^{n} \xi\right)=I\left(\frac{\alpha^{n}}{a} a \xi+\frac{a-\alpha^{n}}{a} 0\right) \geq \frac{\alpha^{n}}{a} I(a \xi)>\alpha^{n} I(\xi)$. Contradiction.

Homogeneity for $a>1$ follows trivially.

Q.E.D.

\section{B.1. Proof of Theorem 1}

By taking $k=0$ in Lemma 1 , the indifference to timing of uncertainty implies that $I(\beta \xi)=\beta I(\xi)$ for any $\xi \in B_{0}(\Sigma, u(X))$. It follows that $I(\beta \xi+k)=$ $\beta I(\xi)+k=I(\beta \xi)+k$ for any $\xi \in B_{0}(\Sigma, u(X))$ and $k \in u(X)$, which means that

$$
I(\zeta+k)=I(\zeta)+k \quad \text { for any } \zeta \in B_{0}(\Sigma, u(X)) \text { and } k \in u(X) .
$$

If $u(X)=\mathbb{R}$, then $I$ satisfies the definition of vertical invariance of Maccheroni, Marinacci, and Rustichini (2004, Definition (iii), p. 18).

Suppose that $u(X)=\mathbb{R}_{+}$and that $\zeta \in B_{0}(\Sigma, u(X))$ and $\zeta+k \in B_{0}(\Sigma, u(X))$ for some $k \in \mathbb{R}$; it follows that $k \geq-\inf \zeta$. If $k<0$, then $I(\zeta)=I((\zeta+k)-$ 
$k)=I(\zeta+k)-k$, where the second equality follows from (13). Thus, $I(\zeta)+$ $k=I(\zeta+k)$ for all $0>k \geq-\inf \zeta$. This equality follows directly from (13) for $k \geq 0$; thus $I$ satisfies the definition of vertical invariance of Maccheroni, Marinacci, and Rustichini (2004, Definition (iii), p. 18).

Hence, $I$ is vertically invariant on $B_{0}(\Sigma, u(X))$ in both cases $u(X)=\mathbb{R}$ and $u(X)=\mathbb{R}_{+}$. By Lemma 23 of Maccheroni, Marinacci, and Rustichini (2004), $I$ is a niveloid and hence is continuous. By Lemma 20 of Maccheroni, Marinacci, and Rustichini (2004), quasiconcavity of $I$ implies its concavity. By Lemma 5 of this paper, $I$ is positively homogeneous. Thus it satisfies the assumptions of Lemma 3.5 of Gilboa and Schmeidler (1989); therefore, there exists a closed and convex set $C \subseteq \Delta(S)$ such that $I(\xi)=\min _{p \in C} \int \xi \mathrm{d} p$ for all $\xi: S \rightarrow \mathbb{R}$.

Q.E.D.

\section{B.2. Proof of Theorem 2}

By Lemma 1, the preference for earlier resolution of uncertainty is equivalent to $I(\beta \xi+k) \geq \beta I(\xi)+k$ for all $\xi \in B_{0}(\Sigma, u(X))$ and $k \in u(X)$. By concavity of $I, I(\beta \xi+k)=I\left(\beta \xi+(1-\beta) \frac{k}{1-\beta}\right) \geq \beta I(\xi)+(1-\beta) I\left(\frac{k}{1-\beta}\right)=$ $\beta I(\xi)+k$.

Q.E.D.

\section{B.3. Proof of Corollary 1}

The proof of Corollary 1 follows from Theorem 2, since the functional $I$ for variational preferences is concave.

Q.E.D.

\section{B.4. Proof of Theorem 3}

By Lemma 25 of Maccheroni, Marinacci, and Rustichini (2006a), it suffices to show that

$$
I(\xi)-I(\zeta) \leq \sup (\xi-\zeta) \text { for all } \xi, \zeta \in B_{0}(\Sigma) .
$$

Fix $\xi \in B_{0}(\Sigma)$ and $k \in \mathbb{R}$ and let $b \in(0,1)$. By concavity, $I(b \xi+k)=I(b \xi+$ $\left.(1-b) \frac{k}{1-b}\right) \geq b I(\xi)+(1-b) I\left(\frac{k}{1-b}\right)$ and, by normalization, $(1-b) I\left(\frac{k}{1-b}\right)=k$. Thus,

$$
I(b \xi+k) \geq b I(\xi)+k \quad \text { for all } \xi \in B_{0}(\Sigma), k \in \mathbb{R} \text {, and } b \in(0,1) .
$$

Observe that $\|b \xi+k-\xi-k\|=\|(1-b) \xi\|=(1-b)\|\xi\|$; therefore, $\lim _{b \rightarrow 1}(b \xi+k)=\xi+k$. Thus, by (14) and by continuity of $I$,

$$
I(\xi+k) \geq I(\xi)+k \quad \text { for all } \xi \in B_{0}(\Sigma) \text { and } k \in \mathbb{R} .
$$

Observe that for all $\xi \in B_{0}(\Sigma)$ and $k \in \mathbb{R}$,

$$
\begin{aligned}
I(\xi+k) \geq I(\xi)+k & =I((\xi+k)-k)+k \\
& \geq I(\xi+k)-k+k=I(\xi+k),
\end{aligned}
$$


where the first inequality follows from (15) applied to $\xi$ and $k$, and the second inequality follows from (15) applied to $\xi+k$ and $-k$. Thus, (16) implies that

$$
I(\xi+k)=I(\xi)+k \quad \text { for all } \xi \in B_{0}(\Sigma) \text { and } k \in \mathbb{R} .
$$

Note that $\xi-\sup (\xi-\zeta)=\xi+\inf (\zeta-\xi) \leq \zeta$; thus

$$
I(\xi)-\sup (\xi-\zeta)=I(\xi-\sup (\xi-\zeta)) \leq I(\zeta),
$$

where the equality follows from (16) applied to $\xi$ and $-\sup (\xi-\zeta)$, and the inequality is a consequence of monotonicity of $I$. Rearranging (18) delivers the conclusion.

Q.E.D.

\section{B.5. Proof of Corollary 2}

(i) Part (i) follows from Theorem 2, since the functional $I$ for confidence preferences is concave.

(ii) Constant relative ambiguity aversion means that $I(\beta \xi)=\beta I(\xi)$ for all $\xi \in B_{0}(\Sigma)$. By Lemma $1, I(\beta \xi+k) \geq \beta I(\xi)+k$ for all $\xi \in B_{0}(\Sigma)$ and $k \in \mathbb{R}$. Thus $I(\beta \xi+k) \geq \beta I(\xi)+k=I(\beta \xi)+k$ for all $\xi \in B_{0}(\Sigma)$ and $k \in \mathbb{R}$, which means that

$$
I(\zeta+k) \geq I(\zeta)+k \quad \text { for all } \zeta \in B_{0}(\Sigma) \text { and } k \in \mathbb{R} .
$$

Suppose, anticipating a contradiction, that $I(\zeta+k)>I(\zeta)+k$ for some $\zeta \in B_{0}(\Sigma)$ and $k \in \mathbb{R}$. Then $I(\zeta+k)>I(\zeta)+k=I((\zeta+k)-k)+k \geq I(\zeta+$ $k)-k+k=I(\zeta+k)$, where the last inequality follows from (19). Contradiction. Thus $I$ satisfies the assumptions of Lemma 3.5 of Gilboa and Schmeidler (1989), so there exists a closed, convex set $C \subseteq \Delta(S)$ with $I(\xi)=\min _{p \in C} \int \xi \mathrm{d} p$ for all $\xi: S \rightarrow \mathbb{R}$.

Q.E.D.

\section{B.6. Proof of the SOEU Case of Theorems 4 and 7}

Let $\Phi:=\phi(u(X))$. Preference for earlier resolution of uncertainty is equivalent to

$$
\int \phi(\beta \xi+k) \mathrm{d} p \geq \phi\left(\beta \phi^{-1}\left(\int \phi(\xi) \mathrm{d} p\right)+k\right)
$$

for all $\xi \in B_{0}(\Sigma, u(X))$ and $k \in u(X)$.

For each $k \in u(X)$, define $F_{k}: \Phi \rightarrow \mathbb{R}$ by $F_{k}(\gamma)=\phi\left(\beta \phi^{-1}(\gamma)+k\right)$. With this notation, (20) becomes $\int F_{k}(\phi(\xi)) \mathrm{d} p \geq F_{k}\left(\int \phi(\xi) \mathrm{d} p\right)$ for all $\xi \in B_{0}(\Sigma$, $u(X))$ and $k \in u(X)$. By letting $\zeta=\phi(\xi)$, this is equivalent to

$$
\int F_{k}(\zeta) \mathrm{d} p \geq F_{k}\left(\int \zeta \mathrm{d} p\right) \text { for all } \zeta \in B_{0}(\Sigma, \Phi) \text { and } k \in u(X) \text {. }
$$


Next, condition (21) is equivalent to convexity of $F_{k}$ for all $k \in u(X)$. To see that, observe that sufficiency follows from Jensen's inequality. Conversely, suppose that (21) holds and find an event $E \in \Sigma$ such that $0<p(E)<1$, denoting $\alpha:=p(E)$. For any $\gamma, \gamma^{\prime} \in \Phi$, condition (21) applied to $\zeta=\gamma_{E} \gamma^{\prime}$ implies that $\alpha F_{k}(\gamma)+(1-\alpha) F_{k}\left(\gamma^{\prime}\right) \geq F_{k}\left(\alpha \gamma+(1-\alpha) \gamma^{\prime}\right)$ for each $k \in u(X)$. By Theorem 88 of Hardy, Littlewood, and Pólya (1952), for each $k \in u(X)$, function $F_{k}$ is convex (for any $k$, the function $F_{k}$ is continuous). An application of Lemmas 3 and 4 leads to the desired conclusions.

Q.E.D.

\section{B.7. Proof of the Klibanoff-Marinacci-Mukerji Case of Theorems 4 and 8}

Let $\Phi:=\phi(\mathbb{R})$. By Lemma 1, preference for earlier resolution of uncertainty is equivalent to

$$
\begin{aligned}
& \int_{\Delta(\Sigma)} \phi\left(\beta \int \xi \mathrm{d} p+k\right) \mathrm{d} \mu(p) \\
& \quad \geq \phi\left(\beta \phi^{-1}\left(\int_{\Delta(\Sigma)} \phi\left(\int \xi \mathrm{d} p\right) \mathrm{d} \mu(p)\right)+k\right)
\end{aligned}
$$

for all $\xi \in B_{0}(\Sigma, u(X))$ and $k \in u(X)$.

For each $k \in u(X)$, define $F_{k}: \Phi \rightarrow \mathbb{R}$ by $F_{k}(\gamma)=\phi\left(\beta \phi^{-1}(\gamma)+k\right)$. With this notation, this condition becomes

$$
\begin{aligned}
& \int_{\Delta(\Sigma)} F_{k}\left(\phi\left(\int \xi \mathrm{d} p\right)\right) \mathrm{d} \mu(p) \geq F_{k}\left(\int_{\Delta(\Sigma)} \phi\left(\int \xi \mathrm{d} p\right) \mathrm{d} \mu(p)\right) \\
& \quad \text { for all } \xi \in B_{0}(\Sigma, u(X)) \text { and } k \in u(X) .
\end{aligned}
$$

By defining $Y:=\left\{\chi: \Delta(\Sigma) \rightarrow \mathbb{R} \mid \chi(p)=\phi\left(\int \xi \mathrm{d} p\right)\right.$ for some $\xi \in B_{0}(\Sigma$, $u(X))\}$, this condition can be rewritten as

$$
\int_{\Delta(\Sigma)} F_{k}(\chi) \mathrm{d} \mu \geq F_{k}\left(\int_{\Delta(\Sigma)} \chi \mathrm{d} \mu\right) \text { for all } \chi \in Y \text { and } k \in u(X) .
$$

Sufficiency follows from Lemmas 3 and 4 and Jensen's inequality. For necessity, observe that under Assumption 1, the above condition is equivalent to

$$
\sum_{j=1}^{m} F_{k}\left(\chi_{j}\right) \mu_{j} \geq F_{k}\left(\sum_{j=1}^{m} \chi_{j} \mu_{j}\right) \quad \text { for all } \chi \in Y \text { and } k \in u(X),
$$

where $Y=\left\{\chi \in \mathbb{R}^{m} \mid \chi_{j}=\phi\left((M \xi)_{j}\right)\right.$ for some $\left.\xi \in(u(X))^{n}\right\}=\left\{\chi \in \mathbb{R}^{m} \mid \chi_{j}=\right.$ $\phi\left(\zeta_{j}\right)$ for some $\left.\zeta \in(u(X))^{m}\right\}$. Taking $\zeta=(a, b, \ldots, b)$ for all $a, b \in u(X)$ ensures that for all $c, d \in \Phi$, the set $Y$ includes all vectors of the form 
$(c, d, \ldots, d)$. Hence, the preference for earlier resolution of uncertainty implies that

$$
F_{k}(c) \mu_{1}+F_{k}(d)\left(1-\mu_{1}\right) \geq F_{k}\left(c \mu_{1}+d\left(1-\mu_{1}\right)\right) \quad \text { for all } c, d \in \Phi .
$$

By Theorem 88 of Hardy, Littlewood, and Pólya (1952), for each $k \in u(X)$, function $F_{k}$ is convex (for any $k$ the function $F_{k}$ is continuous). An application of Lemmas 3 and 4 leads to the desired conclusions.

Q.E.D.

\section{B.8. Proof of Theorem 6}

Define $B:=\beta+\beta^{2}+\cdots+\beta^{T}$. Fix $\xi \in B_{0}(\Sigma, u(X))$ and define $\alpha \in(0,1)$ by $\alpha:=\frac{1}{1+B}$. By normalization and concavity of $I$,

$$
\begin{aligned}
I(\xi) & =I(\alpha(1+B) \xi+(1-\alpha) 0) \\
& \geq \alpha I((1+B) \xi)+(1-\alpha) I(0)=\alpha I((1+B) \xi),
\end{aligned}
$$

so

$$
0 \leq \varepsilon:=(1+B) I(\xi)-I((1+B) \xi) .
$$

To prove the aversion to long-run risk, note that by the argument that leads to expression (11) in the proof of Theorem 3 (with $k=B I(\xi)$ ), we have

$$
I(\xi+B I(\xi)) \geq I(\xi)+B I(\xi) .
$$

Thus, by (22),

$$
I(\xi+B \xi) \leq I(\xi+B I(\xi)),
$$

which, by Lemma 2 , proves the aversion to long-run risk.

To prove the second part of the theorem, note that by Lemma 2, indifference toward long-run risk implies that for any $\xi \in B_{0}(\Sigma, u(X))$,

$$
I(\xi+B I(\xi))=I((1+B) \xi) .
$$

Claim 1. I is positively homogeneous. To see that, note that

$$
\xi+B I(\xi)=\alpha[\xi+B \xi]+(1-\alpha)[I(\xi)+B I(\xi)] .
$$

Let

$$
B_{0}(\Sigma, u(X)) \ni \psi:=\alpha[\xi+B \xi]+(1-\alpha) I(\xi+B \xi)
$$

and note that

$$
\psi=\xi+B I(\xi)-(1-\alpha) \varepsilon .
$$


Note that by quasiconcavity and normalization of $I$ and by (24), we have $I(\psi) \geq I(\xi+B \xi)$. If $\varepsilon>0$, then strict monotonicity implies that $I(\xi+$ $B I(\xi))>I(\psi)$. Thus, for any $\xi \in B_{0}(\Sigma, u(X))$, we have $(1+B) I(\xi)=I((1+$ $B) \xi$ ), which implies positive homogeneity by setting $\zeta:=\frac{1}{\alpha} \xi$ and applying Lemma 5. This concludes the proof of Claim 1.

Claim 2. For any $\xi$ and any $\gamma \in[0,1]$,

$$
I(\gamma x+(1-\gamma) y)=I((1+B) \xi),
$$

where $x:=(1+B) \xi$ and $y:=I((1+B) \xi)$. To prove the claim, observe that, by positive homogeneity,

$$
\xi+B I(\xi)=\alpha x+(1-\alpha) y .
$$

From the normalization assumption, $I(x)=I(y)$, so by indifference to longrun risk and Lemma 2,

$$
I(\alpha x+(1-\alpha) y)=I(\xi+B I(\xi))=I((1+B) \xi) .
$$

Define $f:[0,1] \rightarrow \mathbb{R}$ by $f(\gamma):=I(\gamma x+(1-\gamma) y)-I((1+B) \xi)$ and observe that $f(0)=f(1)=f(\alpha)=0$ for some $0<\alpha<1$. Observe that $f$ is a concave and nonnegative function as a consequence of the concavity of $I$. Suppose that there exists $\gamma \in(0,1)$ with $f(\gamma)>0$. If $\gamma>\alpha$, then $\alpha=\delta \gamma+(1-\delta) 0$ for $\delta=\alpha \gamma^{-1}$. By concavity, $0=f(\alpha) \geq \delta f(\gamma)+(1-\delta) f(0)>0$, contradiction. If $\gamma<\alpha$, then $\alpha=\delta \gamma+(1-\delta) 1$ for $\delta=(1-\alpha)(1-\gamma)^{-1}$. By concavity, $0=$ $f(\alpha) \geq \delta f(\gamma)+(1-\delta) f(1)>0$, contradiction. This concludes the proof of Claim 2.

Claim 3. $I(\xi+k)=I(\xi)+k$ for all $\xi, k$ such that $I(\xi) \geq 0$ and $k>0$. To prove the claim, first assume that $I(\xi)>0$ and let $\gamma$ be defined by

$$
\frac{1-\gamma}{\gamma}=\frac{k}{I(\xi)}
$$

and define

$$
\zeta:=\frac{1}{\gamma(1+B)} \xi .
$$

Then

$$
\xi+k=\gamma(1+B) \zeta+(1-\gamma) I((1+B) \zeta),
$$

so by the above claim, it follows that

$$
\begin{aligned}
I(\xi+k) & =I((1+B) \zeta)=I\left((1+B) \frac{1}{\gamma(1+B)} \xi\right)=\frac{1}{\gamma} I(\xi) \\
& =I(\xi)+\left(\frac{1}{\gamma}-1\right) I(\xi)=I(\xi)+k .
\end{aligned}
$$


By continuity, the above equality implies that $I(\xi+k)=I(\xi)+k$ for all $\xi, k$ such that $I(\xi)=0$ and $k>0$, which concludes the proof of Claim 3 .

Claim 4. $I(\xi+k)=I(\xi)+k$ for all $\xi, k$ such that $I(\xi) \leq 0$ and $k<0$. The proof is analogous.

Claim 5. $I(\xi+k)=I(\xi)+k$ for all $\xi, k$. To prove the claim, fix $\xi$ with $I(\xi) \geq$ 0 and suppose that $k<0$. (The case when $I(\xi) \leq 0$ and $k>0$ is analogous.) First, if $I(\xi+k) \geq 0$, then let $\zeta:=\xi+k$ and note that Claim 3 implies that $I(\zeta+(-k))=I(\zeta)+(-k)$, which is the desired conclusion. Suppose now that $I(\xi+k)<0$. By continuity, there exists $l \in \mathbb{R}$ such that $I(\xi+l)=0$. Let $\zeta:=\xi+l$ and note that by Claim 3 we have that $l=-I(\xi)$. Now, by Claim 4, $I(\xi+k)=I(\zeta+(k-l))=I(\zeta)+(k-l)=0+k+I(\xi)$, which concludes the proof of Claim 5.

To conclude the proof, note that Claim 5 implies that $I$ is vertically invariant. Thus $I$ satisfies the assumptions of Lemma 3.5 of Gilboa and Schmeidler (1989); therefore, there exists a closed and convex set $C \subseteq \Delta(S)$ such that $I(\xi)=\min _{p \in C} \int \xi \mathrm{d} p$ for all $\xi: S \rightarrow \mathbb{R}$.

Q.E.D.

\section{B.9. Proof of Corollary 3}

(i) Define $B:=\beta+\beta^{2}+\cdots+\beta^{T}$. Fix $\xi \in B_{0}(\Sigma, u(X))$ and define $\alpha \in(0,1)$ by $\alpha:=\frac{1}{1+B}$. Since variational preferences are concave, Theorem 6 proves the aversion to long-run risk.

(ii) Define $B:=\beta+\beta^{2}+\cdots+\beta^{T}$. Fix $\xi \in B_{0}(\Sigma, u(X))$ and define $\alpha \in$ $(0,1)$ by $\alpha:=\frac{1}{1+B}$. Since for $u(x)=\mathbb{R}_{+}$, confidence preferences are concave, Theorem 6 proves the aversion to long-run risk.

(iii) Case (iii) follows from cases (i) and (ii) and the fact that a smooth ambiguity preference with concave $\phi$ CARA or CRRA is a variational preference or a confidence preference respectively.

Q.E.D.

\section{B.10. Proof of Theorem 5}

Sufficiency is trivial. For necessity, assume that $\widehat{V}_{t}$ is the representation of $\left\{\succsim_{t, \omega}\right\}$ in terms of ( $\left.W^{\text {disc }}, I, u\right)$ and let $\widetilde{V}_{t}$ be its representation in terms of $\left(W, I^{\mathrm{MEU}}, v\right)$.

Step $1-v=\phi \circ u$. Fix $x_{0}, x_{1}, \ldots, x_{T-1} \in X$ and let $h$ range over $\left(x_{0}, x_{1}, \ldots\right.$, $\left.x_{T-1}, x\right)$ for $x \in X$. Then $\widehat{V}_{T}(\omega, h)=u(x)$, while $\widetilde{V}_{T}(\omega, h)=v(x)$. Because $\widehat{V}_{T}$ and $\widetilde{V}_{T}$ represent the same order $\succsim_{T, \omega}$, they have to be ordinally equivalent; thus, there exists a strictly increasing function $\phi: \mathbb{R} \rightarrow \mathbb{R}$ such that $v(x)=\phi(u(x))$ for all $x \in X$.

Step 2-I $(\xi):=\phi^{-1}\left(\min _{p \in C} \int \phi(\xi) \mathrm{d} p\right)$. Fix $x_{0}, x_{1}, \ldots, x_{T-1} \in X$ and let $h$ range over $\left(x_{0}, x_{1}, \ldots, x_{T-1}, \check{f}_{T}\right)$ for $f \in \mathcal{F}$. Observe that $\widehat{V}_{T-1}(\omega, h)=$ $u\left(x_{T-1}\right)+\beta I(u(f))$, while

$$
\widetilde{V}_{T-1}(\omega, h)=W\left(x_{T-1}, I^{\mathrm{MEU}}(v(f))\right)=W\left(x_{T-1}, \min _{p \in C} \int \phi(u(f)) \mathrm{d} p\right) .
$$


Because $\widehat{V}_{T-1}$ and $\widetilde{V}_{T-1}$ represent the same order, $\succsim_{T-1, \omega}$, they have to be ordinally equivalent; in particular, their restrictions to $\mathcal{F}$ have to be ordinally equivalent. Of course, the restriction of $\widehat{V}_{T-1}$ is ordinally equivalent to $I(u(f))$ and the restriction of $\widetilde{V}_{T-1}$ is ordinally equivalent to $\phi^{-1}\left(\min _{p \in C} \int \phi(u(f)) \mathrm{d} p\right)$. Observe that they coincide on constant acts; for this reason, it is possible to define induced preferences over "utility acts" $B_{0}(\Sigma)$. The representation of this preference induced by $\widehat{V}_{T-1}$ is simply $I$, while the representation induced by $\widetilde{V}_{T-1}$ is $J(\xi):=\phi^{-1}\left(\min _{p \in C} \int \phi(\xi) \mathrm{d} p\right)$. They are both ordinally equivalent, so there exists a strictly increasing function $\psi: \mathbb{R} \rightarrow \mathbb{R}$ such that $I(\xi)=\psi(J(\xi))$ for all $\xi \in \mathcal{B}_{0}(\Sigma)$. In particular, this must hold for all constant utility acts: $I(r)=\psi(J(r))$ for all $r \in \mathbb{R}$. However, $I$ is normalized by assumption, whereas $J$ is normalized by direct verification. Thus, for all $r \in \mathbb{R}$, it must be that $r=I(r)=\psi(J(r))=\psi(r)$; hence, $\psi$ is identity and $I$ is equal to $J$.

Step $3-\phi$ is continuous and concave. Recall that there exists an essential event $E \in \Sigma$. Let $\alpha:=\min _{p \in C} p(E)$ and note that $0<\alpha<1$. The mapping $I$ induces a mapping on the set $\left\{(\bar{k}, \underline{k}) \in \mathbb{R}^{2} \mid \bar{k} \geq \underline{k}\right\}$ by considering utility acts of the form $\bar{k}_{E} \underline{k}$. This induced mapping is $(\bar{k}, \underline{k}) \mapsto \alpha \phi(\bar{k})+(1-\alpha) \phi(\underline{k})$; observe that it inherits quasiconcavity. To show the concavity of $\phi$, for any $k \in$ $\mathbb{R}$, consider the restriction of the quasiconcave mapping $(\bar{k}, \underline{k}) \mapsto \alpha \phi(\bar{k})+(1-$ $\alpha) \phi(\underline{k})$ to the set $(-\infty, k) \times(k, \infty)$. By Theorem 1 of Debreu and Koopmans (1982), the function $\phi$ is continuous on $(k, \infty)$; hence it is continuous on $\mathbb{R}$ in light of the arbitrary choice of $k$. By the proposition of Yaari (1977), $\phi$ is a concave function on $(k, \infty)$; hence it is concave on $\mathbb{R}$ in light of the arbitrary choice of $k$.

Step $4-W(x, d)=\varphi\left(u(x)+\beta \phi^{-1}(d)\right)$. Recall that $\widehat{V}_{T-1}$ and $\widetilde{V}_{T-1}$ are ordinally equivalent on $X \times \mathcal{F}$. For this reason, there exists a strictly increasing function $\varphi: \mathbb{R} \rightarrow \mathbb{R}$ such that

$$
W\left(x_{T-1}, \min _{p \in C} \int \phi(u(f)) \mathrm{d} p\right)=\varphi\left(u\left(x_{T-1}\right)+\beta I(u(f))\right)
$$

for all $x_{T-1} \in X$ and all $f \in \mathcal{F}$. Because $I$ is equal to $J$, it follows that $W\left(x_{T-1}, \phi(I(u(f)))\right)=\varphi\left(u\left(x_{T-1}\right)+\beta I(u(f))\right)$ for all $x_{T-1} \in X$ and all $f \in \mathcal{F}$. This means that $W(x, \phi(\gamma))=\varphi(u(x)+\beta \gamma)$ for all $x \in X$ and all $\gamma \in \mathbb{R}$. Hence, $W(x, d)=\varphi\left(u(x)+\beta \phi^{-1}(d)\right)$ for all $x \in X$ and all $d \in \operatorname{Range}(\phi)$.

Step 5- $\varphi(r)=a \phi(r)+b$. Fix $x_{0}, x_{1}, \ldots, x_{T-2} \in X$ and $x_{T} \in X$ such that $u\left(x_{T}\right)=0$; let $h$ range over $\left(x_{0}, x_{1}, \ldots, x_{T-2}, \check{f}_{T-1}, x_{T}\right)$ for $f \in \mathcal{F}$. Observe that

$$
\begin{aligned}
\widehat{V}_{T-2}(\omega, h) & =u\left(x_{T-2}\right)+\beta I\left(\widehat{V}_{T-1}\left(\left(\omega^{T-1}, \cdot\right), h\right)\right) \\
& =u\left(x_{T-2}\right)+\beta I(u(f)) .
\end{aligned}
$$


Thus, $\widehat{V}_{T-2}$ induces a quasiconcave order on $B_{0}(\Sigma)$ represented by $\xi \mapsto$ $\min _{p \in C} \int \phi(\xi) \mathrm{d} p$. On the other hand,

$$
\begin{aligned}
\widetilde{V}_{T-2}(\omega, h) & =W\left(x_{T-2}, I^{\mathrm{MEU}}\left(\widetilde{V}_{T-1}\left(\left(\omega^{T-1}, \cdot\right), h\right)\right)\right) \\
& =\varphi\left(u\left(x_{T-2}\right)+\beta \phi^{-1}\left(I^{\mathrm{MEU}}\left(\widetilde{V}_{T-1}\left(\left(\omega^{T-1}, \cdot\right), h\right)\right)\right)\right) \\
& =\varphi\left(u\left(x_{T-2}\right)+\beta \phi^{-1}\left(I^{\mathrm{MEU}}(\varphi(u(f)))\right)\right) .
\end{aligned}
$$

Thus, $\widetilde{V}_{T-2}$ induces an order on $B_{0}(\Sigma)$ represented by $\xi \mapsto \min _{p \in C} \int \varphi(\xi) \mathrm{d} p$.

Because $\widehat{V}_{T-2}$ and $\widetilde{V}_{T-2}$ are ordinally equivalent, the mappings $\xi \mapsto$ $\min _{p \in C} \int \phi(\xi) \mathrm{d} p$ and $\xi \mapsto \min _{p \in C} \int \varphi(\xi) \mathrm{d} p$ represent the same quasiconcave order on $B_{0}(\Sigma)$. Both mappings induce mappings on the set $\left\{(\bar{k}, \underline{k}) \in \mathbb{R}^{2} \mid \bar{k} \geq\right.$ $\underline{k}\}$ by considering utility acts of the form $\bar{k}_{E} \underline{k}$. These induced mappings are $(\bar{k}, \underline{k}) \mapsto \alpha \phi(\bar{k})+(1-\alpha) \phi(\underline{k})$ and $(\bar{k}, \underline{k}) \mapsto \alpha \varphi(\bar{k})+(1-\alpha) \varphi(\underline{k})$; observe that they inherit quasiconcavity. Standard arguments from expected utility theory (see, e.g., Theorem 83 of Hardy, Littlewood, and Pólya (1952)) imply that there exist $a>0, b \in \mathbb{R}$ such that $\varphi(r)=a \phi(r)+b$ for all $r \in \mathbb{R}$.

Step 6- $a \phi(r)+b=\phi(r+c)$. Fix $x_{0}, x_{1}, \ldots, x_{T-3} \in X$ and $x_{T} \in X$ such that $u\left(x_{T}\right)=0$; let $h$ range over $\left(x_{0}, x_{1}, \ldots, x_{T}\right)$ for $x_{T-1}, x_{T-2} \in X$. Observe that

$$
\widehat{V}_{T-2}(\omega, h)=u\left(x_{T-2}\right)+\beta u\left(x_{T-1}\right)
$$

and

$$
\widetilde{V}_{T-2}(\omega, h)=a \phi\left(u\left(x_{T-2}\right)+\beta \phi^{-1}\left(a \phi\left(u\left(x_{T-1}\right)\right)+b\right)\right)+b .
$$

They both induce an order on $X \times X$ that has two additive representations:

$$
(x, y) \mapsto u(x)+\beta u(y)
$$

and

$$
(x, y) \mapsto u(x)+\beta \phi^{-1}(a \phi(u(y)))+b .
$$

By the uniqueness of additively separable representations, since the first component utility is identical in both representations, this implies that $\phi^{-1}(a \phi(u(y))+b)=u(y)+c$, so $a \phi(u(y))+b=\phi(u(y)+c)$. This implies that $a \phi(r)+b=\phi(r+c)$ for all $r \in \mathbb{R}$.

Step $7-\varphi=\phi$ or $\phi(r)=d a^{r / c}+\frac{b}{1-a}$. Since $\phi$ is continuous and concave, $\chi:=\phi^{-1}$ is a convex function. Thus, both $\phi$ and $\chi$ have at most countably many points of nondifferentiability. Focus attention on the complement of this set. If $a=1$ and $c \neq 0$, then $\phi^{\prime}(r)=\phi^{\prime}(r+c)$, which implies that $\phi$ is affine and this concludes the proof, since $I^{\mathrm{MEU}}$ is invariant to affine transformations. If $a=1$ and $c=0$, then $b=0$ and this concludes the proof. Suppose $a \neq 1$. If 
$c=0$, then $(1-a) \phi(r)=b$, which is a contradiction, since $\phi$ is nonconstant. Thus, $a \neq 1$ and $c \neq 0$. In this case then, the most general solution to $a \phi(r)+$ $b=\phi(r+c)$ is $\phi(r)=d a^{r / c}+\frac{b}{1-a}$; see, for example, Polyanin and Manzhirov (2008).

Q.E.D.

\section{REFERENCES}

Anscombe, F., AND R. Aumann (1963): "A Definition of Subjective Probability," The Annals of Mathematical Statistics, 34, 199-205. [1059]

BANSAL, R., AND A. YARON (2004): "Risks for the Long Run: A Potential Resolution of Asset Pricing Puzzles," Journal of Finance, 59, 1481-1509. [1041]

BANSAL, R., D. KIKU, AND A. YARON (2012): "An Empirical Evaluation of the Long-Run Risks Model for Asset Prices," Critical Finance Review, 1, 183-221. [1041]

BARILlaS, F., L. HANSEN, AND T. SARGENT (2009): “Doubts or Variability?” Journal of Economic Theory, 144, 2388-2418. [1040]

BENIGNO, P., AND S. NisTiCò (2009): "International Portfolio Allocation Under Model Uncertainty," Working Paper 14734, National Bureau of Economic Research. [1040]

BlaCKWELl, D. (1953): "Equivalent Comparisons of Experiments," The Annals of Mathematical Statistics, 24, 265-272. [1040]

CAMERER, C., AND M. WeBER (1992): "Recent Developments in Modeling Preferences: Uncertainty and Ambiguity," Journal of Risk and Uncertainty, 5, 325-370. [1039]

Cerreia-Vioglio, S., F. Maccheroni, M. Marinacci, AND L. Montrucchio (2011): "Uncertainty Averse Preferences," Journal of Economic Theory, 146, 1275-1330. [1044,1045, 1052]

Chateauneuf, A., AND J. H. FARo (2009): "Ambiguity Through Confidence Functions," Journal of Mathematical Economics, 45, 535-558. [1044]

CHEN, H., N. JU, AND J. MiAO (2009): "Dynamic Asset Allocation With Ambiguous Return Predictability,” Working Paper. [1040]

CHEN, Z., AND L. EPSTEIN (2002): "Ambiguity, Risk, and Asset Returns in Continuous Time," Econometrica, 70, 1403-1443. [1040,1049]

CHEW, S., AND L. EPSTEIN (1990): "Nonexpected Utility Preferences in a Temporal Framework With an Application to Consumption-Savings Behaviour," Journal of Economic Theory, 50, 54-81. [1056]

CHEw, S. H., AND L. EPSTEIN (1989): "The Structure of Preferences and Attitudes Towards the Timing of the Resolution of Uncertainty," International Economic Review, 30, 103-117. [1041, 1045,1057]

Collard, F., S. MuKerJI, K. Sheppard, AND J. M. TAllon (2011): "Ambiguity and the Historical Equity Premium,” Working Paper. [1040]

Debreu, G., AND T. Koopmans (1982): "Additively Decomposed Quasiconvex Functions," Mathematical Programming, 24, 1-38. [1069]

DeKel, E., B. LipMAN, AND A. Rustichini (2001): "Representing Preferences With a Unique Subjective State Space," Econometrica, 69, 891-934. [1059]

DrechSLER, I. (2009): "Uncertainty, Time-Varying Fear, and Asset Prices," Working Paper. [1040]

DUfFIE, D., AND L. EPSTEIN (1992): "Stochastic Differential Utility,” Econometrica, 60, 353-394. $[1039,1041,1054]$

EllsberG, D. (1961): "Risk, Ambiguity, and the Savage Axioms," Quarterly Journal of Economics, 75, 643-669. [1039]

EPSTEIN, L. G., AND M. SCHNEIDER (2003a): "IID: Independently and Indistinguishably Distributed," Journal of Economic Theory, 113, 32-50. [1045,1049]

$1048]$ (2003b): "Recursive Multiple-Priors," Journal of Economic Theory, 113, 1-31. [1045, 
EPStein, L. G., AND T. WANG (1994): "Intertemporal Asset Pricing Under Knightian Uncertainty," Econometrica, 62, 283-322. [1040]

EpsteIN, L. G., AND S. ZIN (1989): "Substitution, Risk Aversion, and the Temporal Behavior of Consumption and Asset Returns: A Theoretical Framework," Econometrica, 57, 937-969. $[1040,1045,1053]$

(1991): "Substitution, Risk Aversion, and the Temporal Behavior of Consumption and Asset Returns: An Empirical Analysis," Journal of Political Economy, 99, 263. [1040]

EPSTEIN, L. G., E. FARHI, AND T. STRZALECKI (2013): "How Much Would You Pay to Resolve Long-Run Risk?” Working Paper. [1043]

Ergin, H., AND F. Gul (2009): "A Theory of Subjective Compound Lotteries," Journal of Economic Theory, 144, 899-929. [1040,1044]

ERgin, H., AND T. SARVER (2010): "Hidden Actions and Preferences for Timing of Resolution of Uncertainty," Working Papert. [1059]

GILBOA, I., AND D. SCHMEIDLER (1989): "Maxmin Expected Utility With Non-Unique Prior," Journal of Mathematical Economics, 18, 141-153. [1039,1041,1044,1063,1064,1068]

GRANT, S., AND B. POLAK (2013): "Mean-Dispersion Preferences and Constant Absolute Uncertainty Aversion," Journal of Economic Theory (forthcoming). [1045]

GRANT, S., A. KAJII, AND B. POLAK (1998): "Intrinsic Preference for Information,” Journal of Economic Theory, 83, 233-259. [1045]

(2000): "Temporal Resolution of Uncertainty and Recursive Non-Expected Utility Models," Econometrica, 68, 425-434. [1041,1045,1057]

HALEVY, Y. (2007): "Ellsberg Revisited: An Experimental Study," Econometrica, 75, 503-536. [1039]

HANANY, E., AND P. KLIBANOFF (2007): “Updating Multiple-Prior Preferences,” Theoretical Economics, 2, 261-298. [1048]

(2009): "Updating Ambiguity Averse Preferences," The B.E. Journal of Theoretical Economics, 9 (1), Article 37. [1048]

HANSEN, L., J. HeAton, AND N. Li (2008): “Consumption Strikes Back? Measuring Long-Run Risk," Journal of Political Economy, 116, 260-302. [1041]

HANSEN, L. P., AND T. J. SARGENT (2001): "Robust Control and Model Uncertainty," American Economic Review, 91, 60-66. [1042,1044,1055]

HARDY, G., J. LiTTLEWOOD, AND G. PÓlYA (1952): Inequalities. Cambridge: Cambridge University Press. [1065,1066,1070]

HAYASHI, T. (2005): "Intertemporal Substitution, Risk Aversion and Ambiguity Aversion," Economic Theory, 25, 933-956. [1053,1058,1059]

HAYASHI, T., AND J. MIAO (2011): "Intertemporal Substitution and Recursive Smooth Ambiguity Preferences," Theoretical Economics, 6, 423-472. [1059]

IluT, C. (2012): "Ambiguity Aversion: Implications for the Uncovered Interest Rate Parity Puzzle," American Economic Journal: Macroeconomics, 4 (3), 33-65. [1040]

JU, N., AND J. MiAO (2012): "Ambiguity, Learning, and Asset Returns," Econometrica, 80, 559-591. [1040]

Karantounias, A. G., L. P. Hansen, And T. J. SARgent (2012): "Managing Pessimistic Expectations and Fiscal Policy," Theoretical Economics, 8, 193-231. [1040]

Keynes, J. (1921): A Treatise on Probability. London: MacMillan. [1039]

Kinlstrom, R., AND L. Mirman (1974): "Risk Aversion With Many Commodities," Journal of Economic Theory, 8, 361-388. [1056]

KLESHCHELSKI, I., AND N. VINCENT (2009): "Robust Equilibrium Yield Curves,” Working Paper. [1040]

KLIBANOFF, P., M. MARINACCI, AND S. MuKerJI (2005): "A Smooth Model of Decision Making Under Ambiguity," Econometrica, 73, 1849-1892. [1040,1044,1045,1059]

(2009): "Recursive Smooth Ambiguity Preferences," Journal of Economic Theory, 144, 930-976. [1048,1060] 
KLIBANOFF, P., AND E. OZDENOREn (2007): "Subjective Recursive Expected Utility," Economic Theory, 30, 49-87. [1053]

KNIGHT, F. (1921): Risk, Uncertainty and Profit. Boston: Houghton Mifflin Company. [1039]

KoopmAns, T. (1960): "Stationary Ordinal Utility and Impatience," Econometrica, 28, 287-309. [1053]

KREPS, D. (1979): “A Representation Theorem for 'Preference for Flexibility'," Econometrica, 47, 565-577. [1059]

KREPS, D., AND E. PORTEUS (1978): “Temporal Resolution of Uncertainty and Dynamic Choice Theory," Econometrica, 46, 185-200. [1039,1040,1045,1046,1049,1053,1056]

LAZRAK, A. (2004): "Generalized Stochastic Differential Utility and Preference for Information," The Annals of Applied Probability, 14, 2149-2175. [1053]

LI, M., AND A. TORnELL (2008): "Exchange Rates Under Robustness: An Account of the Forward Premium Puzzle,” Working Paper. [1040]

Maccheroni, F., M. MARINACCI, AND A. RUSTIChini (2004): "Ambiguity Aversion, Malevolent Nature, and the Variational Representation of Preferences," Working Paper. [1062,1063]

(2006a): "Ambiguity Aversion, Robustness, and the Variational Representation of Preferences," Econometrica, 74, 1447-1498. [1040,1044,1063]

(2006b): "Dynamic Variational Preferences," Journal of Economic Theory, 128, 4-44. $[1045,1048]$

MACHINA, M. (1989): "Dynamic Consistency and Non-Expected Utility Models of Choice Under Uncertainty," Journal of Economic Literature, 27 (4), 1622-1668. [1048]

Maenhout, P. (2004): "Robust Portfolio Rules and Asset Pricing," Review of Financial Studies, 17, 951-983. [1040]

NAU, R. (2006): “Uncertainty Aversion With Second-Order Utilities and Probabilities," Management Science, 52, 136-145. [1044]

NeILson, W. S. (1993): "Ambiguity Aversion: An Axiomatic Approach Using Second Order Probabilities," Working Paper. [1040,1044]

Neyman, J., AND E. SCOTT (1948): “Consistent Estimates Based on Partially Consistent Observations," Econometrica, 16, 1-32. [1049]

PolyANIN, A., AND A. MANZHIROV (2008): Handbook of Integral Equations. Boca Raton, FL: Chapman \& Hall/CRC Press. [1071]

QuigGin, J. (1982): “A Theory of Anticipated Utility,” Journal of Economic Behavior and Organization, 3, 323-343. [1058]

SARIN, R., AND P. P. WAKKER (1998): "Dynamic Choice and Nonexpected Utility,” Journal of Risk and Uncertainty, 17, 87-119. [1048]

(2000): “Cumulative Dominance and Probabilistic Sophistication," Mathematical Social Sciences, 40, 191-196. [1045]

SchmeIDLER, D. (1989): "Subjective Probability and Expected Utility Without Additivity," Econometrica, 57, 571-587. [1039,1044,1045,1059]

SEgAL, U. (1987): "The Ellsberg Paradox and Risk Aversion: An Anticipated Utility Approach," International Economic Review, 28, 175-202. [1044]

(1990): "Two-Stage Lotteries Without the Reduction Axiom," Econometrica, 58, 349-377. [1045]

Selden, L. (1978): “A New Representation of Preferences Over 'Certain $\times$ Uncertain' Consumption Pairs: The 'Ordinal Certainty Equivalent' Hypothesis,” Econometrica, 46, 1045-1060. [1056]

SEO, K. (2009): "Ambiguity and Second-Order Belief," Econometrica, 77, 1575-1605. [1044,1059]

SinisCALCHI, M. (2011): "Dynamic Choice Under Ambiguity," Theoretical Economics, 6, 379-421. [1048]

SKIADAS, C. (1998): "Recursive Utility and Preferences for Information," Economic Theory, 12, 293-312. [1053]

SPENCE, M., AND R. ZeCKHAUSER (1972): "The Effect of the Timing of Consumption Decisions and the Resolution of Lotteries on the Choice of Lotteries," Econometrica, 40, 401-403. [1040] 
STRZALECKI, T. (2011): "Axiomatic Foundations of Multiplier Preferences," Econometrica, 79, 47-73. [1044,1051,1052]

TAllarini, T. D. (2000): "Risk-Sensitive Real Business Cycles," Journal of Monetary Economics, 45, 507-532. [1040]

WEIL, P. (1989): "The Equity Premium Puzzle and the Risk-Free Rate Puzzle," Journal of Monetary Economics, 24, 401-421. [1040]

(1990): “Nonexpected Utility in Macroeconomics," Quarterly Journal of Economics, 105, 29-42. [1040]

YAARI, M. E. (1977): “A Note on Separability and Quasiconcavity,” Econometrica, 45, 1183-1186. [1069]

(1987): "The Dual Theory of Choice Under Risk," Econometrica, 55, 95-115. [1058]

Harvard University, Cambridge, MA 02138, U.S.A.; tomasz_strzalecki@ harvard.edu.

Manuscript received October, 2010; final revision received October, 2012. 